\title{
Role of proNGF/p75 signaling in bladder dysfunction after spinal cord injury
}

\author{
Jae Cheon Ryu, ${ }^{1}$ Katharine Tooke, ${ }^{2}$ Susan E. Malley, ${ }^{2}$ Anastasia Soulas, ${ }^{1}$ Tirzah Weiss, ${ }^{1}$ Nisha Ganesh, ${ }^{1}$ Nabila Saidi, ${ }^{1}$ \\ Stephanie Daugherty, ${ }^{3}$ Uri Saragovi, ${ }^{4}$ Youko Ikeda, ${ }^{5}$ Irina Zabbarova, ${ }^{5}$ Anthony J. Kanai, ${ }^{5}$ Mitsuharu Yoshiyama, ${ }^{6}$ \\ H. Francis Farhadi, ${ }^{7}$ William C. de Groat, ${ }^{3}$ Margaret A. Vizzard, ${ }^{2}$ and Sung Ok Yoon ${ }^{1}$
}

\begin{abstract}
'Department of Biological Chemistry and Pharmacology, Ohio State University, Columbus, Ohio, USA. Department of Neurological Sciences, Larner College of Medicine, University of Vermont, Burlington, Vermont, USA. ${ }^{3}$ Department of Pharmacology and Chemical Biology, University of Pittsburgh, Pittsburgh, Pennsylvania, USA. ${ }^{4}$ Department of Pharmacology and Therapeutics, McGill University, Montreal, Quebec, Canada. ${ }^{5}$ Department of Medicine, University of Pittsburgh, Pittsburgh, Pennsylvania, USA. ${ }^{6}$ Department of Urology, University of Yamanashi Graduate School of Medical Science, Chuo, Japan. ${ }^{7}$ Department of Neurological Surgery, Ohio State University, Columbus, Ohio, USA.
\end{abstract}

\begin{abstract}
Loss of bladder control is a challenging outcome facing patients with spinal cord injury (SCI). We report that systemic blocking of pro-nerve growth factor (proNGF) signaling through p75 with a CNS-penetrating small-molecule p75 inhibitor resulted in significant improvement in bladder function after $\mathrm{SCl}$ in rodents. The usual hyperreflexia was attenuated with normal bladder pressure, and automatic micturition was acquired weeks earlier than in the controls. The improvement was associated with increased excitatory input to the spinal cord, in particular onto the tyrosine hydroxylase-positive fibers in the dorsal commissure. The drug also had an effect on the bladder itself, as the urothelial hyperplasia and detrusor hypertrophy that accompany SCI were largely prevented. Urothelial cell loss that precedes hyperplasia was dependent on p75 in response to urinary proNGF that is detected after SCI in rodents and humans. Surprisingly, death of urothelial cells and the ensuing hyperplastic response were beneficial to functional recovery. Deleting p75 from the urothelium prevented urothelial death, but resulted in reduction in overall voiding efficiency after $\mathrm{SCl}$. These results unveil a dual role of proNGF/p75 signaling in bladder function under pathological conditions with a CNS effect overriding the peripheral one.
\end{abstract}

\section{Introduction}

The function of the micturition reflex is to store urine and then to completely empty urine (i.e., void) at socially appropriate times. Supralumbar spinal cord injury (SCI) results in significant urinary bladder dysfunction leading to life-threatening urinary retention and renal failure without appropriate management (1). SCI rostral to the lumbosacral spinal cord disconnects the brain from the spinal cord, resulting in (a) discoordination between the urinary bladder and external urethral sphincter (i.e., detrusor sphincter dyssynergia [DSD]); (b) inability to inhibit the storage circuits; (c) incomplete activation of voiding circuits; and (d) voiding dysfunction. Over time, the urinary bladder becomes "overactive" (active at low and high bladder volumes), producing small, intermittent contractions of the bladder and unwanted leakage (urinary incontinence) with prominent DSD but highly inefficient voiding (1). Impaired bladder emptying, which results in urinary retention with high bladder pressure, is a significant problem in the care of individuals with SCI (1). The underlying mechanisms that contribute to voiding dysfunction following SCI are due to dramatic changes in the properties of C-fiber bladder afferents (2). There are no drugs approved for the treatment of SCI-induced bladder dysfunction. It is impera-

Conflict of interest: The authors have declared that no conflict of interest exists. Submitted: October 3, 2017; Accepted: February 8, 2018

Reference information: / Clin Invest. 2018;128(5):1772-1786.

https://doi.org/10.1172/JCI97837. tive to identify targets that can enhance elimination reflexes and reduce morbidity following SCI.

Nerve growth factor (NGF) has for some time been a focus for treating various urological conditions, such as overactive bladder and interstitial cystitis/painful bladder syndromes, as it was known to contribute to bladder overactivity (3). In particular, an increase in NGF levels was reported in the urine after SCI as well as in overactive bladder and interstitial cystitis/painful bladder syndromes $(3,4)$, prompting studies to block its action as a way to improve overall bladder function (5). Whether the site of NGF action is only at the urinary bladder or also in the spinal cord is, however, unclear. Previous studies demonstrated that intrathecal delivery of an NGF-blocking antibody attenuates abnormal urethral pressure increases that occur during voiding (i.e., DSD) in SCI animals (5), and sequestering urinary NGF with TrkA-Fc decreased both nonvoiding contractions and overall voiding frequency under cystitis conditions (6).

p75 is a receptor for the neurotrophins NGF, BDNF, and NT3/4. Although first discovered as a survival-promoting receptor for neurotrophins during nervous system development, recent advances in the field clearly demonstrate that p75 conversely induces apoptosis and degeneration under pathological conditions that increase its expression (7). Under these pathological conditions, the preferred ligands that activate p75 are proneurotrophins that are secreted without undergoing intracellular processing. Thus, the current view is that mature neurotrophins activate the complex of cognate Trk receptors and $\mathrm{p} 75$, promoting 
survival and differentiation, whereas proneurotrophins bind p75 selectively and with a higher affinity, inducing apoptosis.

Although p75 expression has previously been identified in micturition reflex pathways, few studies have examined the potential role $(\mathrm{s})$ of p75 in urinary bladder reflexes $(8,9)$. The functional role of $\mathrm{p} 75$ has been addressed in lower urinary tract reflexes in the context of urinary bladder inflammation $(8,9)$. Previous studies demonstrated that $\mathrm{p} 75$ blockade at the level of the urinary bladder increased voiding frequency in control rats and further increased voiding frequency in rats treated with cyclophosphamide (CYP) to induce cystitis (9). Thus, one function of proNGF/p75 interactions may be to reduce bladder activity or to offset increased voiding frequency induced by CYP-induced cystitis (9). The contribution(s) of proneurotrophins (e.g., proNGF) to micturition reflex function have not been explored in the context of health, injury, or disease. Thus, determination of the roles of proNGF/p75 signaling in normal micturition circuitry and urinary bladder morphology may provide additional insights into their contributions to urinary bladder dysfunction after SCI. We hypothesized that proNGF/p75 signaling in the periphery as well as in the CNS regulates bladder dysfunction after SCI. In this study, we have used preclinical models and human tissues to evaluate the contributions and roles of proNGF and p75 to urothelial cell apoptosis and urinary bladder morphology after SCI. In addition, we evaluated the functional contributions of proNGF/p75 signaling to micturition reflexes in spinal-intact and SCI mice using (a) conditional deletion of p75 from urothelial cells and (b) pharmacological blockade with the small molecule LM11A-31 $(10,11)$. Our results suggest that p75 signaling plays important roles in micturition under spinal-intact and SCI conditions in both the CNS and the PNS. Our study of the mechanisms by which proNGF/p75 signaling contributes to bladder dysfunction after SCI will help facilitate rapid development of LM11A-31 or its analog as a viable therapeutic agent to treat bladder dysfunction in the near future.

\section{Results}

ProNGF is detected in the urine in rodents and humans within hours of $S C I$. We subjected urine samples collected at different times after SCI to Western blotting using a proNGF-specific antibody as well as a pan-NGF antibody to detect both mature and proNGF. While mature NGF was not detectable at the time points tested, proNGF was detected in the urine as early as 1 hour after injury, and its levels remained high up to 7 hours after injury (Figure 1, A and B). The lack of mature NGF in the urine was confirmed by mouse mature NGF-specific ELISA (data not shown). A similar time course of urinary proNGF was obtained using mouse proNGF-specific ELISA, which detected urinary proNGF levels in the range of 6.2-10.7 ng/ mg of proteins at 1-7 hours after injury (Figure 1C). The proNGF reactive band was indeed proNGF, as indicated by immunoprecipitation with a monoclonal 27/21 NGF antibody followed by blotting with a proNGF antibody (Figure 1D). It should be noted that SCIinduced increases in urinary proNGF were not limited to mice or the type of SCI: proNGF was detected within 1-7 hours after injury after contusion injuries in mice, and also in rats and humans (Figure $1, E-G)$. The amount of urinary proNGF in 2 human patients was 1 and $46 \mathrm{pg} / \mathrm{mg}$ of proteins, respectively, based on a humanspecific proNGF ELISA. No proNGF was detected in control, unin- jured patient urine. Together, these results suggest that proNGF is a major form of NGF appearing in the urine after SCI in rodents and humans, and the released proNGF is likely to elicit similar responses in mammals after SCI.

NGF expression has been detected in the bladder tissue itself, and its RNA levels were reported to increase after SCI and CYPinduced cystitis (12). In agreement with this report, NGF was detected in both the urothelium and the detrusor muscle in the naive, uninjured state (Supplemental Figure 1A; supplemental material available online with this article; https://oi.org/10.1172/ JCI97837DS1). At 7 hours after injury, when urinary proNGF levels were high (Figure 1), NGF immunoreactivity was significantly reduced in both the urothelium and the detrusor muscle in comparison with the naive sample (Supplemental Figure 1, A and B). In agreement with immunofluorescence data, overall proNGF levels were reduced in the urothelium at 1-4 hours after injury, with a gradual increase until 5 days after injury based on Western blotting (Supplemental Figure 1C). In the detrusor muscle, proNGF levels were increased at 3-5 days after injury (Supplemental Figure 1D). These results suggest that urinary proNGF is likely to have been released from the urothelium and detrusor muscle.

We next asked whether the expression of its signaling receptor, p75, is also similarly regulated in the urothelium and detrusor muscle. p75 was detected in both the urothelium and the detrusor muscle, with a significantly greater expression in the detrusor muscle than in the urothelium based on Western blotting (Supplemental Figure 2A). A similar expression pattern was demonstrated with Trk receptors, with a majority of Trk receptors detected in the detrusor muscle and minimal expression in the urothelium. It is noted that although we suspect the signal observed reflects that from TrkA, we cannot confirm this since we used a pan-Trk antibody here.

We next examined with immunohistochemistry which cells in the bladder express p75 both in the naive state and after SCI. In the naive state, p75 immunoreactivity was detected mainly among the urothelial umbrella cells, colocalizing with uroplakin, a marker for these cells (Supplemental Figure 2, B and C). In addition, $\mathrm{p} 75^{+}$sensory nerve fibers that project to the urothelium were also detected (Supplemental Figure 2B, arrows), as published (8, 9). In the detrusor muscle, an increase in p75 immunoreactivity was detected at 7 hours after injury and persisted up to 5 days after injury (Supplemental Figure 2B). Based on the morphology, this staining most probably represents nerve fiber projections to the muscle, which demonstrated an overall increase in p75 expression after injury. Sortilin, a coreceptor for p75 in binding proNGF, was also expressed both in the urothelium and the muscle (data not shown). In the urothelium, the patterns of p75 expression changed greatly after injury: p75 immunoreactivity was detectable in umbrella cells lining the bladder lumen at 7 hours and 1 day after injury, with some p75 immunoreactivity appearing to label dying cells that were being sloughed off from the urothelial layer (Supplemental Figure 2B). Umbrella cells were reported to undergo desquamation as early as 2 hours after injury, with almost complete loss by 1 day after injury after SCI (13). The loss of umbrella cells was followed by a hyperplastic response of the urothelial basal cells at 3 days after injury, during which p $75^{+}$cells appeared as rows that follow the contour of the hyperplastic urothelial layer (Supplemental Figure 2B), with many of them expressing a mitotic 
A

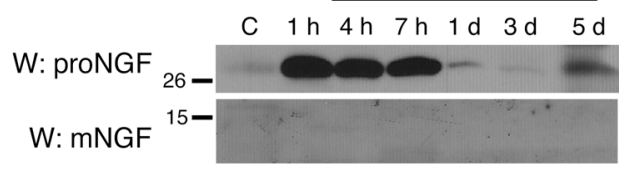

B

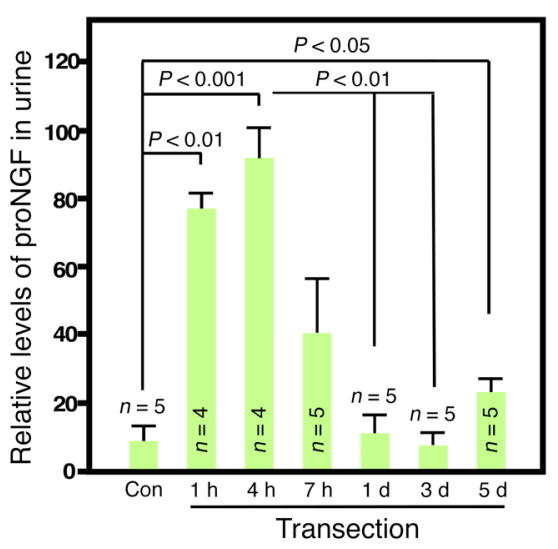

C

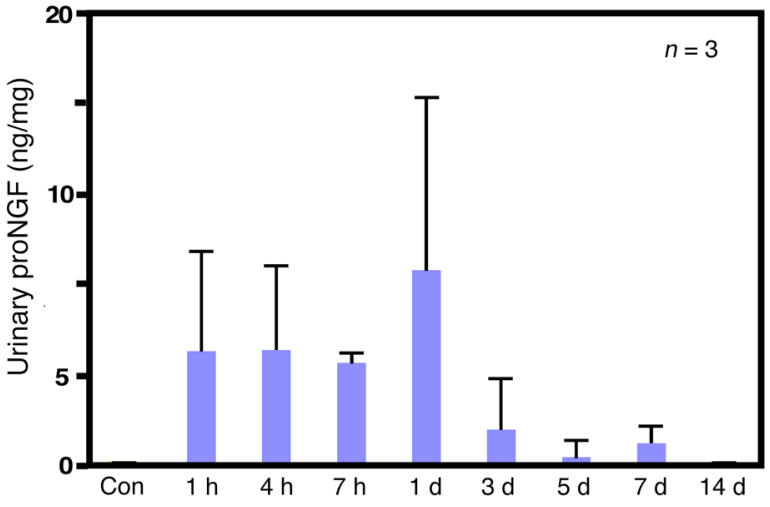

D

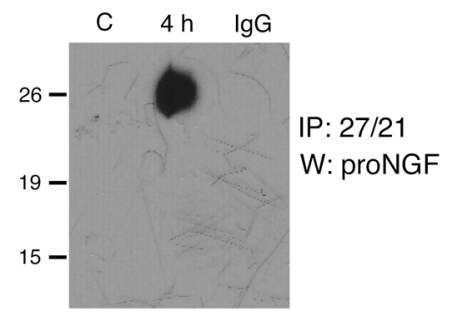

$\mathbf{E}$

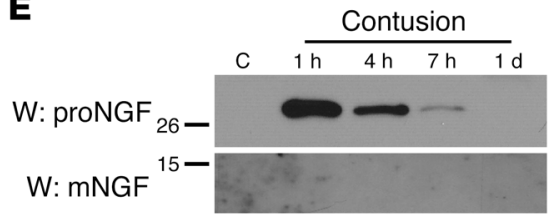

$\mathbf{F}$

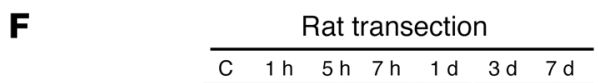

W: proNGF 26

$\begin{array}{llllll}1 \mathrm{~h} & 5 \mathrm{~h} & 7 \mathrm{~h} & 1 \mathrm{~d} & 3 \mathrm{~d} & 7 \mathrm{~d}\end{array}$

W: $\mathrm{mNGF} 15-$

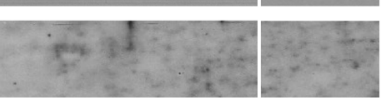

G
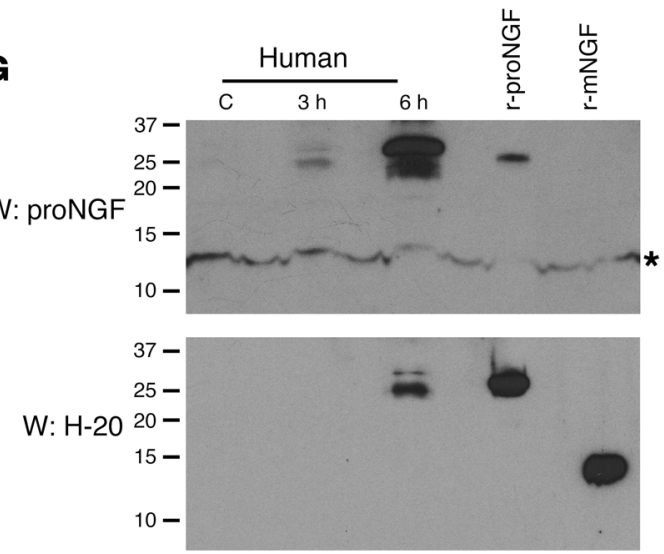

Figure 1. ProNGF is released into the urine in rodents and humans within hours of SCI. (A) ProNGF is released into the urine almost immediately after spinal cord transection in mice. The blots were probed with anti-proNGF and pan-NGF antibodies. There was little mature NGF detectable in the urine at these time points. (B) Quantification of proNGF by Western blotting. Within-subject comparison was significant at $P=0.003$ based on repeated measures of 1-way ANOVA after Greenhouse-Geisser correction, which resulted in $P<0.01$. Subsequent pairwise comparisons were made using Bonferroni correction. (C) Quantification of proNGF detected in mouse urine by proNGF-specific ELISA. Note that mature NGF was not detected by mature NGF-specific ELISA. (D) Immunoprecipitation/Western analyses of urine samples illustrate that the bands detected with proNGF antibody in A are indeed proNGF. Mouse urine samples were immunoprecipitated with 27/21 mature NGF antibody and probed with proNGF antibody. (E) ProNGF is detected in the urine after contusion injuries in mice. (F) ProNGF is present in the urine after spinal cord transection in rats. Mature NGF was not detected by mature NGF-specific ELISA. (G) ProNGF was also detected in human urine after SCI. The upper blot was probed with proNGF-specific antibody (*artifact), while the lower blot was probed with pan-NGF antibody ( $\mathrm{H}-20$ ). Note that recombinant proNGF and mature NGF were included as controls. Based on human proNGF-specific ELISA, the amount of proNGF was 1 and $46 \mathrm{pg} / \mathrm{mg}$ for 3 and 6 hours after injury, respectively. Note that 3 - and 6-hour urine samples were collected from 2 different individuals. ELISA assays also failed to detect mature NGF as in Western blotting. C, urine from healthy control without SCI.

marker, phospho-histone 3 (pH3, Supplemental Figure 2C). After an injury or infection that results in the loss of umbrella cells, the basal cells of the urothelium are known to undergo regeneration, thus replenishing the lost layer (14). At 5 days after injury, after the hyperplastic response had subsided, p75 immunoreactivity was detected in the basal cell layer that abuts the laminin-positive staining (Supplemental Figure 2B and Supplemental Figure 2C), and the cells were also dividing as indicated by $\mathrm{BrdU}$ and $\mathrm{pH} 3$ immunoreactivity (Supplemental Figure 2C). These results suggest that p 75 signaling, perhaps in response to the urinary proNGF released after the injury, plays a role in the loss of umbrella cells and regeneration of the urothelium. p75 is responsible for umbrella cell apoptosis in response to urinary proNGF after SCI. We next asked whether the loss of umbrella cells after SCI is due to apoptosis by performing TUNEL reactions. TUNEL reactions on injured bladder tissues revealed a robust apoptotic response mostly in uroplakin-positive and $p 75^{+}$umbrella cells (Figure 2A). Based on the number of TUNEL ${ }^{+}$umbrella cells, the apoptotic response was most robust at 7 hours after injury, and then subsided rapidly to the background level by 3 days after injury (Figure 2B). To determine whether p75 is involved in inducing the apoptosis of umbrella cells, we subjected $\mathrm{p} 75^{\mathrm{KO}}$ mice to SCI. Remarkably, umbrella cell apoptosis was completely blocked in $\mathrm{p} 75^{\mathrm{KO}}$ mice at 7 hours after injury to 3 days after injury (Figure 2B). 
A

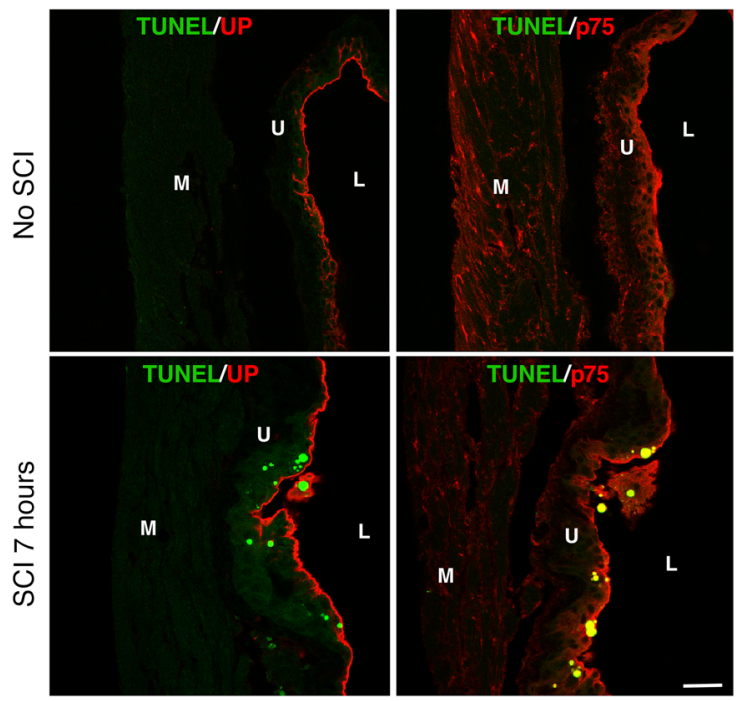

Figure 2. p75 is responsible for umbrella cell apoptosis in response to urinary proNGF after SCI. (A) Uroplakin-positive (UP+) umbrella cells are the major cell types that undergo apoptosis at 7 hours after SCI. The bladders were processed for TUNEL reaction and subsequently stained with UP or p75 antibodies. Urothelial and muscle layers of the bladder wall are indicated by $\mathrm{U}$ and $\mathrm{M}$, respectively, and the bladder lumen is indicated by L. Scale bar: $150 \mu \mathrm{m}$. (B) p75 is responsible for umbrella cell apoptosis after $\mathrm{SCl}$ as indicated by the lack of TUNEL+ cells in the $\mathrm{p} 75^{\mathrm{KO}}$. The data were analyzed using repeated measures of 2-way ANOVA after GreenhouseGeisser correction, which resulted in $P<0.001$ comparing the vehicle and LM11A-31 treatments and the WT and p75-null mice. Subsequent pairwise comparisons were made using Bonferroni correction. (C) Intravesical instillation of proNGF blocking antibody blocked umbrella cell apoptosis completely at 7 hours after injury. The data were analyzed by Student's $t$ tests.
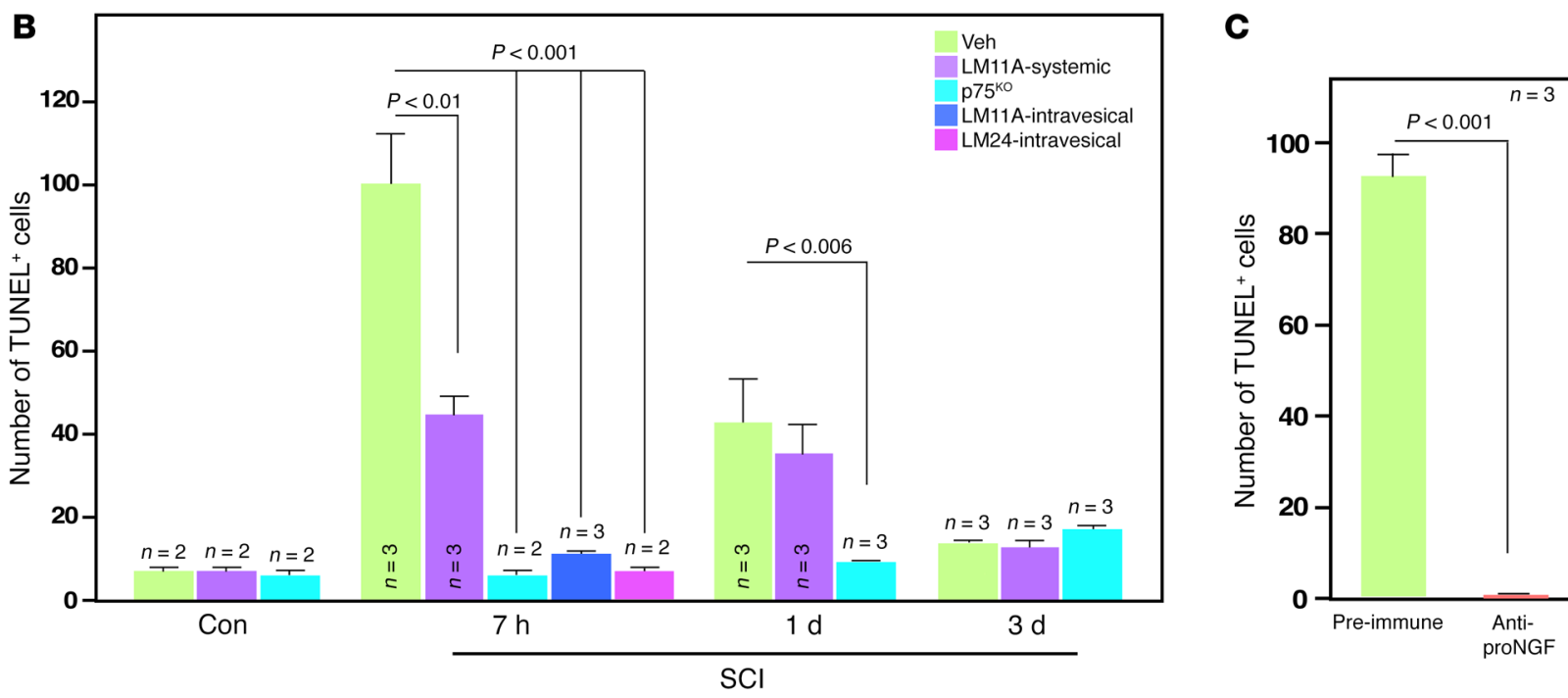

These results suggest that $\mathrm{p} 75$ is involved in inducing umbrella cell apoptosis after SCI. After SCI in $\mathrm{p} 75^{\mathrm{KO}}$ mice, proNGF was detected in the urine at levels similar to those detected in WT SCI mice (data not shown).

We next determined whether blocking proNGF signaling with LM11A-31, which inhibits proNGF action through p75 in vitro and in vivo $(10,11)$, will inhibit umbrella cell apoptosis. Indeed, systemic administration of LM11A-31 via oral gavage at 4 hours after injury reduced the number of $\mathrm{TUNEL}^{+}$urothelial cells at 7 hours after injury by $55 \%$ (Figure $2 \mathrm{~B}$ ). LM11A-31 had no effect at 1 day after injury, in agreement with a significant drop in urinary proNGF levels detected by Western blotting (Figure 1B). When LM11A-31 was delivered immediately after SCI via intravesical instillation, apoptosis of umbrella cells at 7 hours after injury was completely blocked (Figure 2B), suggesting that it is proNGF in the urine that is inducing apoptosis. To confirm whether urinary proNGF is indeed acting as an apoptosis factor, we instilled intravesically either a proNGF-blocking antibody or LM11A-24, immediately after SCI. LM11A-24 is capable of interfering with proNGF binding to p75 to the same extent as
LM11A-31 (10), but fails to cross the blood-brain barrier, and was thus presumed unlikely to cross the urothelial barrier. LM11A-24 as well as proNGF-blocking antibody, but not the control antibody, completely inhibited the apoptosis at 7 hours after injury (Figure 2, B and C). Together, these results indicate that it is the proNGF released into the urine that activates p 75 on the surface of umbrella cells, thereby initiating apoptosis.

In seeking additional support for the critical role of p75 signaling in umbrella cell apoptosis, we examined the morphology of the luminal surface of mice instilled with LM11A-31 and $\mathrm{p} 75^{\mathrm{KO}}$ mice via scanning electron microscopy analysis. Without injury, the luminal surface of the bladder exhibited the well-characterized polyhedral morphology with ridges that demarcate cell boundaries (Figure 3A, blue lines and arrows; ref. 15). At 7 hours after injury, however, the surface morphology was drastically altered, with loss of the characteristic junctional ridges (Figure 3A). By 2 days after injury, umbrella cells were regenerated, but they were smaller in size than those without injury, although they established polyhedral morphology (Figure 3A, red arrows). With intravesical instillation of LM11A-31 immediately after SCI, however, 
A
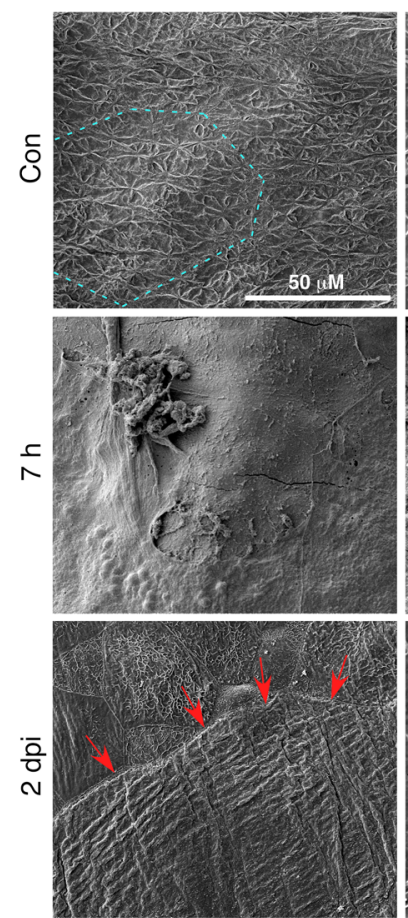

C
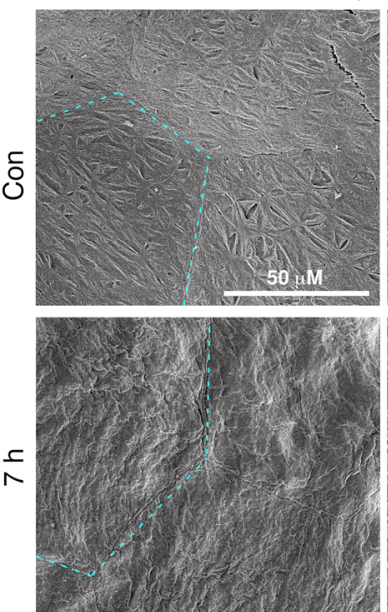

Vehicle
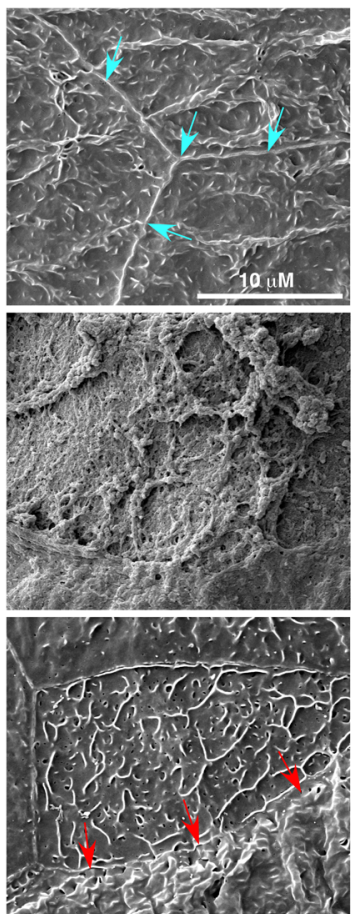

$\mathrm{p} 75^{\mathrm{KO}}$
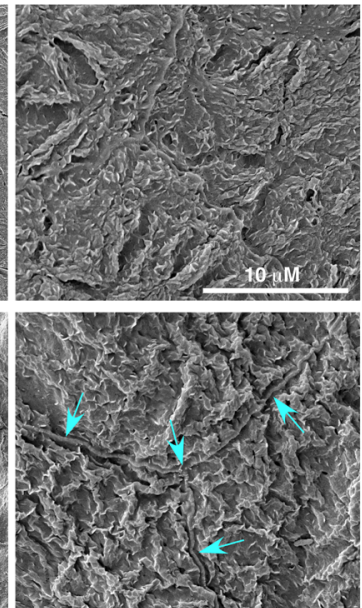

B
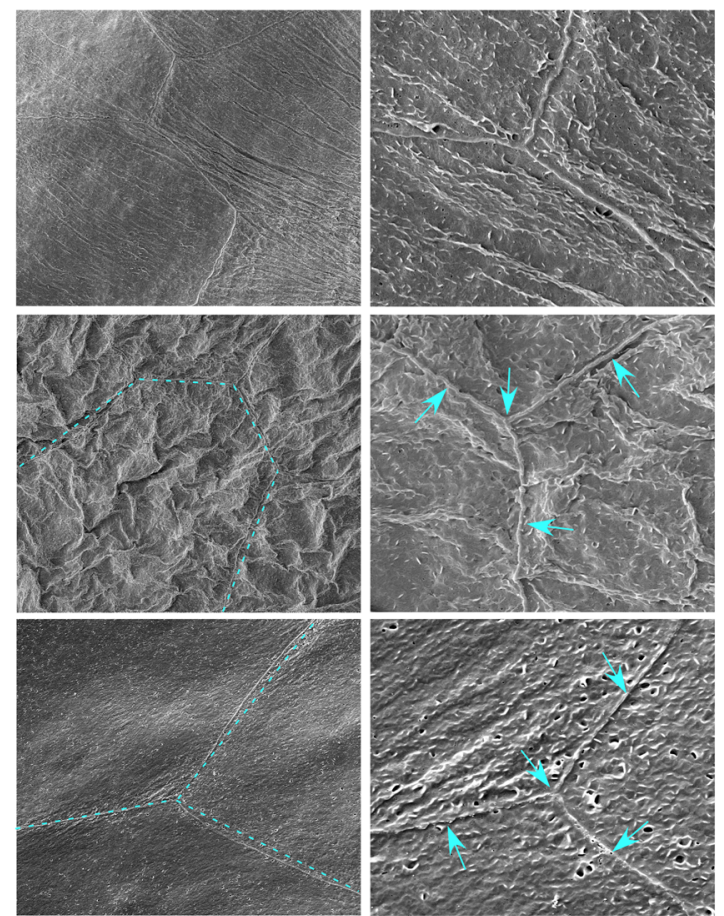

D

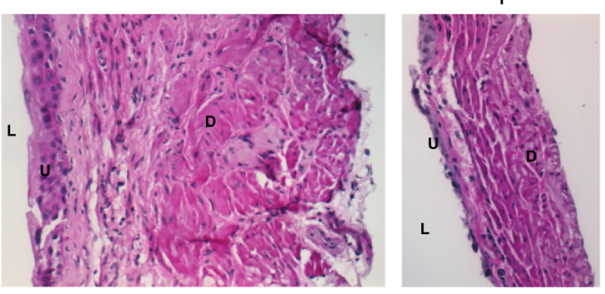

$10 \mathrm{dpi}$

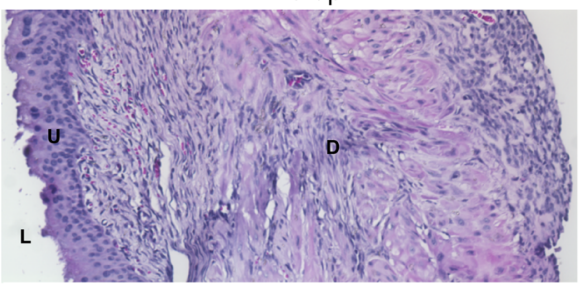

1 dpi + LM11A-31

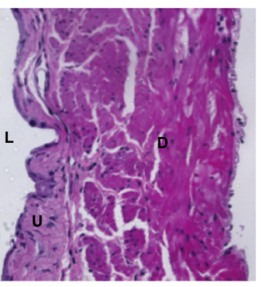

10 dpi + LM11A-31

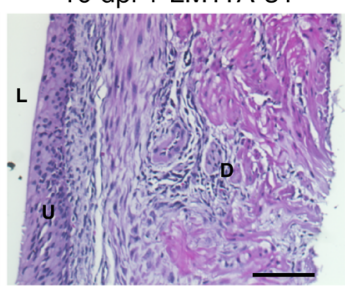

Figure 3. Hexagonal morphology of umbrella cells is preserved in p $75^{\mathrm{Ko}}$ mice and with LM11A-31 administration after SCI. (A) Without injury, the luminal surface of the bladder exhibited the well-characterized polyhedral morphology with ridges that demarcate cell boundaries (blue lines and arrows; ref. 15). At 7 hours after injury, however, the surface morphology was drastically altered, with loss of the characteristic junctional ridges. By 2 days postinjury (dpi) umbrella cells were regenerated, but they were smaller in size than those without injury, although they established polyhedral morphology (red arrows). (B) With intravesical instillation of LM11A-31 immediately after SCI, however, umbrella cell loss was completely blocked, with intact polyhedral ridges at both 7 hours and 2 days after injury (blue lines and arrows). (C) At 7 hours after injury, the luminal surface of the bladder from p75 ${ }^{\mathrm{KO}}$ mice was visually indistinguishable from that of the control bladder. Blue dotted lines mark the hexagonal boundaries of umbrella cells. Higher-magnification images illustrate the cell boundaries between neighboring cells (arrows). (D) H\&E staining of the control and LM11A-31-treated bladders. Note the preservation of the urothelium at 1 day after injury with LM11A-31, while very few urothelial cells are visible in vehicle-treated bladder. LM11A-31 also prevented chronic urothelial hyperplasia and detrusor hypertrophy of the bladder at 10 days after injury. The bladder morphology appears similar to that of the uninjured control bladder. Urothelial and muscle layers of the bladder wall are indicated by $\mathrm{U}$ and $\mathrm{M}$, respectively, and the bladder lumen is indicated by L. Scale bar: 250 $\mu \mathrm{m}$. Experiments were performed 3 times, and representative ones are shown.

umbrella cell loss was completely blocked with intact polyhedral ridges at both 7 hours and 2 days after injury (Figure 3B, blue lines and arrows). Also in $\mathrm{p} 75^{\mathrm{KO}}$ mice at 7 hours after injury, the luminal surface remained almost identical to uninjured control tissue with distinct polyhedral junctional ridges (Figure 3C, blue lines and arrows). The effect of LM11A-31 was also prolonged, as revealed by H\&E histology at 10 days after injury, where urothelial hyperplasia and detrusor hypertrophy were largely prevented (Figure 3D). These results together suggest that proNGF/p75 signaling is responsible for the loss of umbrella cells after SCI. 
A

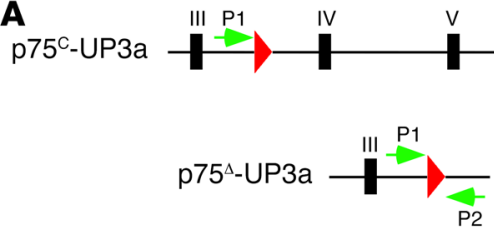

C $\mathrm{p} 75^{\mathrm{C}}-\mathrm{UP} 3 \mathrm{a}$

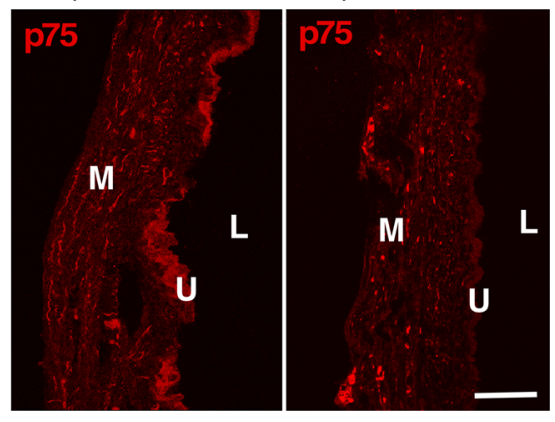

B

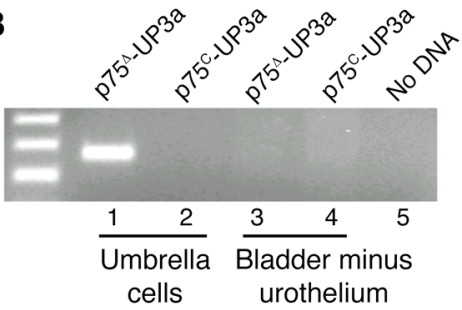

D

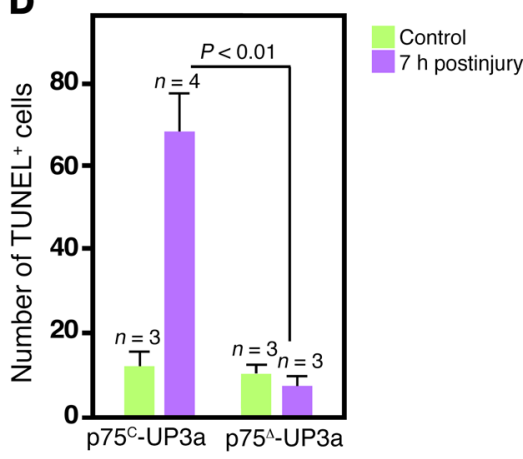

Figure 4. Selective deletion of p75 among umbrella cells results in complete block of umbrella cell apoptosis. (A) Diagram of the targeting vectors in $\mathrm{p} 75^{\wedge}-U P 3 a$ and $p 75^{\circ}-U P 3 a$ mice. Red triangles represent loxP sites, and green $\mathrm{P} 1$ and $\mathrm{P} 2$ arrows represent PCR primers used in $\mathbf{B}$. (B) Selective deletion of p75 in urothelial cells. Urothelial cells from tamoxifen-treated $p 75^{\wedge}$-UP3a and $p 75^{c}$-UP3a mice were scraped into a tube, and the isolated genomic DNA was subjected to PCR using P1 and P2 primers. The primers generate PCR product only when Cre is activated. Note that the PCR band is present only in umbrella cells and not in the bladder minus urothelium in $\mathrm{p} 75^{\Delta}$-UP3a mice, indicating a selective p75 deletion in the urothelium. (C) p75 is not detected in umbrella cells (U), while it is clearly present in the muscle (M). L, bladder lumen. Scale bar: $150 \mu \mathrm{m}$. (D) Umbrella cell apoptosis was completely blocked in p $75^{\perp}-$ UP3a compared with that in $\mathrm{p} 75^{\mathrm{c}}$-UP3a mice.

Selective deletion of p75 among umbrella cells influences micturition. p75 is also expressed in nerve fibers located in the lamina propria below the urothelium and extending into the umbrella cell layer in the urothelium (9). Because this neuronal p75 could contribute to the changes in the umbrella cells after SCI, we generated a conditional p75 knockout mouse line in which p 75 is deleted only among umbrella cells by crossing $\mathrm{p} 75^{\mathrm{n} / \mathrm{l}}$ mice with an inducible uroplakin 3a (UP3a)-CreGFP/ERT2 (16). p75 $5^{\mathrm{f} / \mathrm{l}}$ mice have been described (17). The experimental line, UP3a-CreGFP/ERT2+/$\mathrm{p} 75^{\mathrm{H} / \mathrm{fl}}$, and a control line, UP3a-CreGFP/ERT2 $2^{+-} \mathrm{p} 75^{-/}$, will be referred to as p75 $5^{\wedge}-\mathrm{UP} 3 \mathrm{a}$ and $\mathrm{p} 75^{\mathrm{c}}$-UP3a, respectively, for brevity (Figure 4A). To verify the selective deletion of p75 among umbrella cells and not in other cell types of the bladder, urothelial cells from tamoxifen-treated $\mathrm{p} 75^{\wedge}$-UP3a and $\mathrm{p} 75^{\mathrm{C}}$-UP3a mice were isolated, and their genomic DNA was subjected to PCR reactions using P1 and P2 primers (Figure 4A), which will yield a product only when Cre is activated. The bladder minus urothelium from both genotypes was also used as controls. Indeed, a PCR band was detected only from urothelial cells, and not from the bladder minus urothelium, in p75 $5^{\wedge}-\mathrm{UP} 3 \mathrm{a}$ mice (Figure 4B). The PCR band was also absent in the urothelial cells as well as the bladder minus urothelium from p $75^{c}$-UP3a mice. Similarly, p 75 immunoreactivity was detectable in the innervating fibers in the detrusor muscle, but was absent in the urothelium of $\mathrm{p} 75^{\wedge}$-UP3a mice, while it was present in both layers in p $75^{\mathrm{c}-U P 3 a}$ mice (Figure $4 \mathrm{C}$ ). These results illustrate that p75 is selectively deleted only among urothelial cells in p $75^{\Delta}$ -
UP3a mice. When p75c-UP3a mice were subjected to SCI, the number of TUNEL ${ }^{+}$ apoptotic cells increased at 7 hours after injury, while it remained at the basal level in p75^-UP3a mice (Figure 4D). These results suggest that it is p75 signaling in the urothelium that is responsible for inducing umbrella cell apoptosis.

The urothelium serves as an important permeability barrier, but also exhibits sensory and signaling abilities by releasing many neuromodulators and expressing their receptors, such as nicotinic, muscarinic, tachykinin, adrenergic, bradykinin, and transient receptor potential (TRP) vanilloid receptors (1). These properties allow the urothelium to regulate the excitability and release of neurotransmitters from adjacent capsaicin-sensitive, unmyelinated afferent nerves in the bladder. We thus asked whether protecting the urothelium from disruption that normally accompanies SCI would influence voiding function. Since anesthesia influences voiding function, urodynamic parameters were measured in freely moving conscious mice as published (18). Surprisingly, p75^-UP3a mice exhibited increased voiding frequency with reduced intermicturition intervals and increased bladder pressures compared with naive p $75^{\mathrm{c}}$-UP3a mice (Figure 5 , A-E). The overall voiding efficiency, however, remained similar between the 2 genotypes. This is consistent with a previous study in which pharmacological blockade of NGF binding to p75 increased voiding frequency in control rats and those with CYPinduced cystitis (8). After SCI, however, p75 $5^{\wedge}$-UP3a mice exhibited worse bladder function than the control with an increase in intermicturition intervals and infused volumes, and an overall $32 \%$ reduction in voiding efficiency (Figure 5, A-E). We interpret these results as suggesting that $\mathrm{p} 75$ signaling in the urothelium contributes to micturition in both naive and injured conditions. More importantly, these results suggest that the normal turnover of the urothelium that involves umbrella cell apoptosis plays a protective role for bladder function after SCI.

The phenotype of $\mathrm{p}^{\mathrm{A}} \mathrm{-}-\mathrm{UP} 3 \mathrm{a}$ mice is opposite to that of naive $\mathrm{TrpV} 4^{\mathrm{KO}}$, which showed reduced voiding frequency with increased intermicturition intervals (19). TrpV4 is one of the Trp family of receptors that is expressed in umbrella cells as well as the intermediate and basal cells of the urothelium (20). We thus hypothesized that p75 signaling counteracts $\mathrm{TrpV} 4$ signaling in the urothelium. The hypothesis was tested in primary mouse urothelial cells that express $\mathrm{TrpV} 4$ and respond to agonist addition by increasing calcium influx $(21,22)$. In response to its selective agonist GSK1016790A, $\mathrm{Ca}^{2+}$ flux was increased (Figure 5F), and was dependent on extracellular calcium (data not shown). Preincubation with $10 \mathrm{ng} / \mathrm{ml}$ proNGF 30 minutes before GSK1016790A 

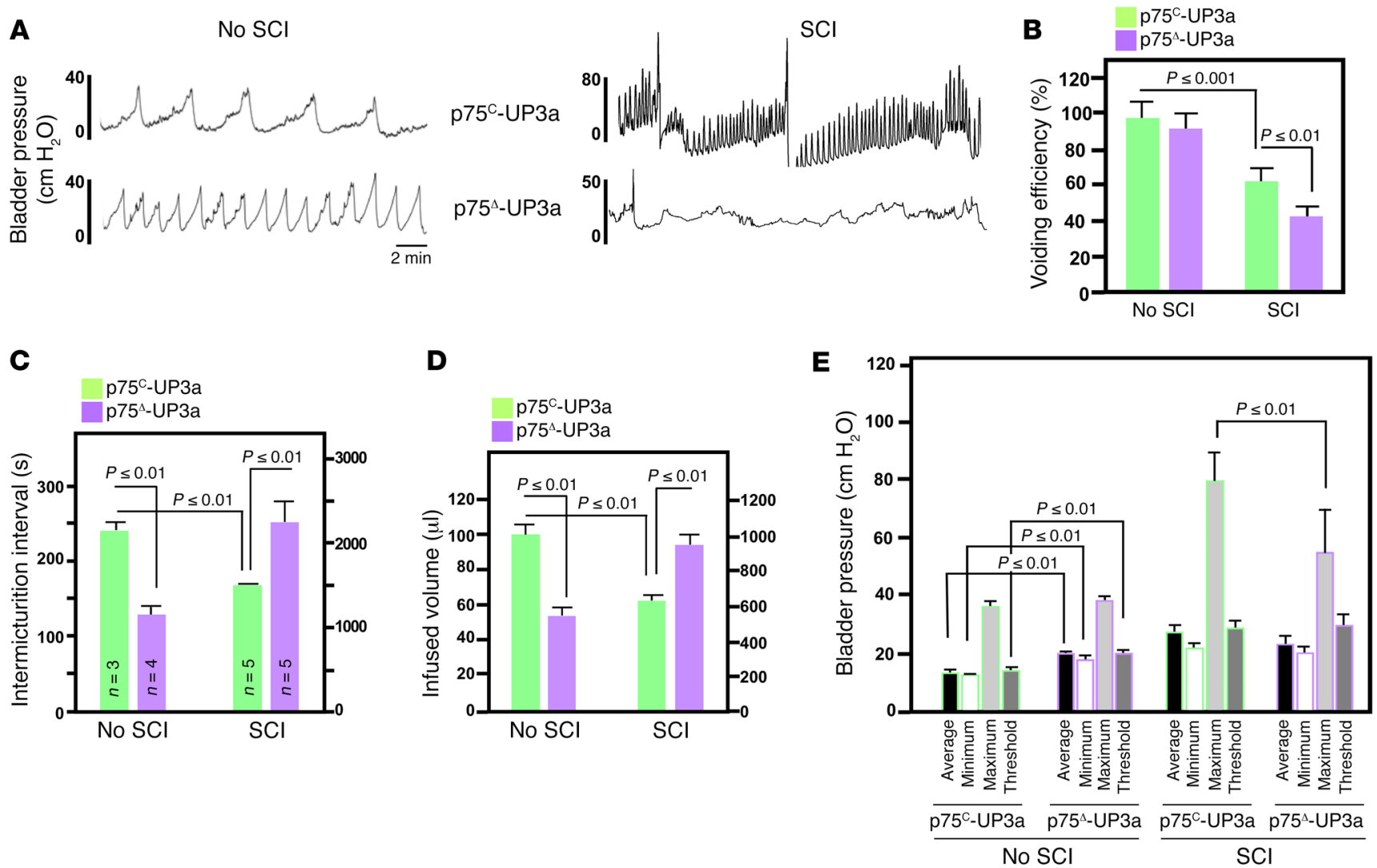

$\mathbf{F}$
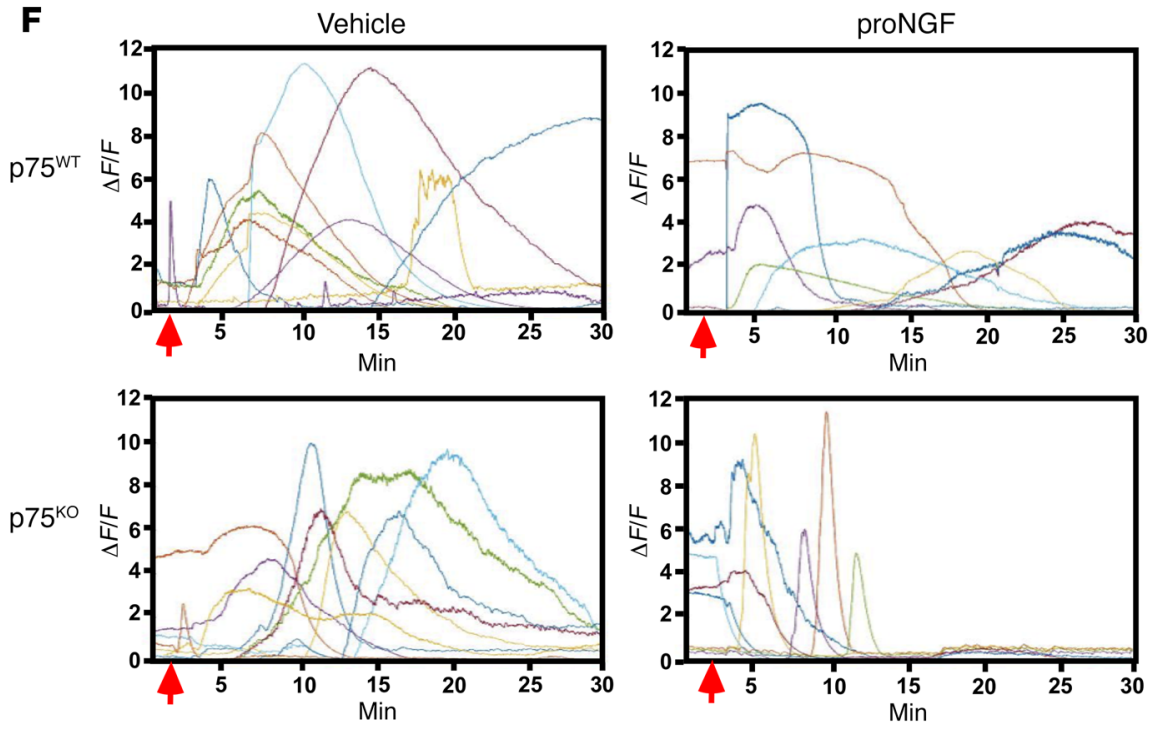

H
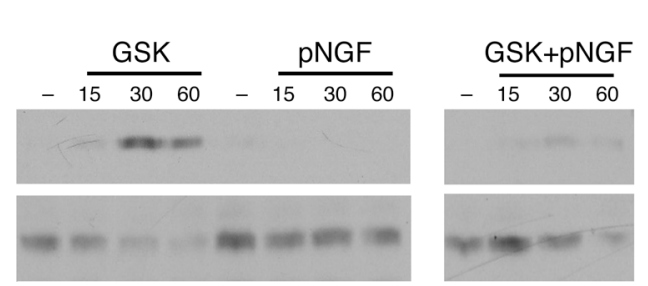

GSK+pNGF
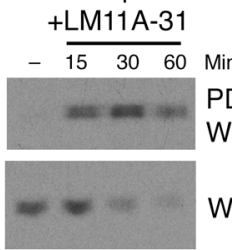

PD: Avidin

W: TrpV4

W: TrpV4

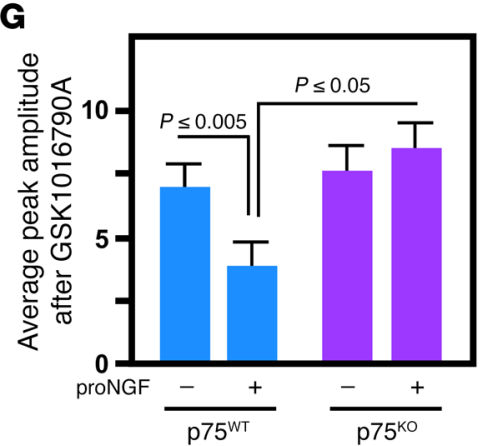


Figure 5. Selective deletion of p75 among umbrella cells influences micturition. (A) Representative recordings of intravesical pressure during continuous intravesical infusion of saline and an open urethral outlet in conscious, unrestrained $\mathrm{p} 75^{\circ}-\mathrm{UP} 3 \mathrm{a}$ and $\mathrm{p} 75^{\wedge}-\mathrm{UP} 3 a$ mice with no SCl and $\mathrm{SCl}$ (2 weeks). (B) Overall voiding efficiency in no-SCI p75 ${ }^{\Delta}-\mathrm{UP3} a$ and p75'-UP3a mice was similar, although after SCl, p75 ${ }^{\wedge}-U P 3 a$ mice exhibited worse bladder function than p75-UP3a mice with a 32\% drop in voiding efficiency $\left(n=3-5\right.$ ). (C and D) Under no-SCI condition, p75 ${ }^{\wedge}$-UP3a mice had significantly decreased infused volumes (IVs) that induced micturition and intermicturition intervals compared with those observed in p75-UP3a mice. SCI significantly reduced intermicturition intervals (C) and IVs (D) in p75'-UP3a mice, and these effects were reversed in p $75^{\wedge}$-UP3a mice. Note that left $y$ axes represent no $\mathrm{SCl}$, while right $y$ axes represent $\mathrm{SCl}$ groups. (E) No-SCI p75^-UP3a mice exhibited significantly increased average, minimum, and threshold bladder pressures compared with control, with no change in maximum micturition pressure. Comparisons among groups were made using ANOVA. When $F$ ratios exceeded the adjusted critical value $(P \leq 0.0125)$, Bonferroni's multiple-comparisons test was used to compare means among groups. (F) ProNGF/p75 signaling negatively influences TrpV4-mediated $\mathrm{Ca2}^{+}$flux in primary mouse urothelial cells. Urothelial cells were incubated with $10 \mathrm{ng} / \mathrm{ml}$ of proNGF or vehicle for 30 minutes before Fluo-4 AM loading and GSK1016790A addition at $50 \mathrm{nM}$ (red arrows; $n=4$ independent experiments). (C) Quantification of the average peak amplitude of $\triangle F / F$. Comparisons among groups were made using ANOVA and pairwise comparisons were made by Tukey's multiple-comparisons test ( $3-8$ cells were included per treatment). (H) GSK1016790A facilitates cell-surface targeting of TrpV4 in HEK293T cells, which is inhibited by proNGF. ProNGF-mediated inhibition is lifted with LM11A-31 preincubation.

addition attenuated the $\mathrm{Ca}^{2+}$ flux significantly as shown by $57 \%$ reduction in the peak amplitude, suggesting that proNGF signaling antagonizes TrpV4 activation by GSK1016790A (Figure 5, F and G). In support, GSK1016790A-mediated $\mathrm{Ca}^{2+}$ flux was unaffected in $\mathrm{p} 75^{\mathrm{KO}}$ urothelial cultures (Figure $5, \mathrm{~F}$ and $\mathrm{G}$ ). It should be noted that measurements of the area under the curve of $\mathrm{Ca}^{2+}$ flux failed to show any significant differences among the 4 groups (data not shown). In order to understand the mechanism by which proNGF inhibits TrpV4 activity, we tested whether cell-surface expression of TrpV4 is regulated by its agonist, and whether proNGF modulates it in 293T cells that were transfected with p75, sortilin, and TrpV4. GSK1016790A addition increased the trafficking of TrpV4 to the cell surface, with its peak at 30 minutes (Figure $5 \mathrm{H}$ ). The addition of proNGF prior to GSK1016790A application reduced the amount of TrpV4 targeted to the cell membrane without influencing its transport kinetics. Addition of LM11A-31 along with proNGF in turn counteracted the effect of proNGF addition, with increased levels of TrpV4 detected on the cell surface. ProNGF alone failed to have any effect. These results together suggest that urinary proNGF can influence TrpV4 activity in the urothelium, thereby regulating voiding frequency.

Systemic LM11A-31 significantly improves overall bladder function. Surprised at our unexpected results with $p 75^{\wedge}$-UP3a mice, we decided to test the effect of systemic LM11A-31 administration, since we have reported that proNGF is the major form of NGF that is released into the cerebrospinal fluid after CNS injuries including SCI $(23,24)$, potentially influencing the central and peripheral circuits that control micturition. In addition, LM11A-31 crosses the brain-spinal cord barrier efficiently after SCI (11). For these experiments, LM11A-31 was orally delivered at $100 \mathrm{mg} / \mathrm{kg}$ beginning 4 hours after injury, and once daily thereafter for 4 weeks.
LM11A-31 administration resulted in significant improvement in voiding function with the overall voiding efficiency reaching that of spinal-intact mice, and the bladder capacity and pressure also returned to levels as under naive conditions (Figure 6, A-C). Accordingly, the infused volume and intermicturition intervals were also reduced by $50 \%$ and $55 \%$, respectively (Figure 6, D and E). Bladder weight was also reduced with LM11A-31 treatment (69-74 $\pm 4 \mathrm{mg}$ for spine-intact mice; $123 \pm 3 \mathrm{mg}$ for SCI-vehicle mice; $87 \pm 4 \mathrm{mg}$ for SCI-LM11A-31). These changes culminated in a greater number of mice recovering automatic micturition earlier than the vehicle control after SCI (Figure 6F). As we have shown that LM11A-31 targets p 75 in vivo (11), these results together suggest that proNGF signaling through p75 in the spinal cord contributes significantly to disruption of voiding function after SCI, and blocking proNGF action through p75 can lead to significant improvement in bladder control after SCI.

Systemic LM11A-31 increases glutamatergic inputs onto tyrosine hydroxylase-positive neurons after SCI. It was reported that NGF regulates synaptic organization in the sympathetic system, where TrkA promoted while $\mathrm{p} 75$ inhibited postsynaptic specialization (25). We next asked whether the micturitional improvement observed with LM11A-31 was also accompanied by synaptic changes. As the first step, we assessed the number of $\mathrm{c}-\mathrm{fos}^{+}$cells in the area of the dorsal commissure (DCM) of L6 spinal cords, choosing these in particular since their numbers were shown to increase with bladder distention among preganglionic neurons $(26,27)$. At 28 days after injury, the number of $\mathrm{c}-$ fos $^{+}$cells increased at least 2-fold, while LM11A-31 administration reduced the number to that in no-SCI control (Figure 7, A and B). This is reminiscent of reports that capsaicin treatment reduced the number of $\mathrm{c}^{-\mathrm{fos}^{+}}$cells (27) after SCI, and increased voiding efficiency (28), suggesting that capsaicin-sensitive C-fiber afferents are involved in initiation of detrusor-sphincter dyssynergia and/or detrusor hyperreflexia. We thus looked for proteins that are expressed in small-diameter dorsal root ganglion (DRG) neurons that project to the DCM of L6/ S1 spinal cords, and whose expression overlaps with $\mathrm{p} 75$. A protein that fit the criteria was tyrosine hydroxylase (TH) (Figure 7, $\mathrm{C}$ and D): Small-diameter neurons expressed both $\mathrm{p} 75$ and TH in L6/S1 DRG regardless of SCI, and their projections were detected in the DCM of the L6/S1 spinal cords. These $\mathrm{TH}^{+}$neurons have been characterized as the ones representing the cutaneous lowthreshold mechanoreceptors (29). After SCI, many of these fiber projections appeared lost (Figure 7D), and instead a distinct group of neurons emerged with clear cell soma morphology in the area of the DCM, expressing both p75 and TH. In accordance with these data, modulating dopamine production has recently been shown to influence micturition after SCI (30).

We asked whether LM11A-31 modulated synaptic inputs onto these $\mathrm{TH}^{+}$neurons in the DCM. Since glutamate is the main neurotransmitter released by afferent fibers, we first measured the extent of glutamatergic inputs to the L6 spinal cord by quantifying the number of vGlut1 $1^{+}$synaptic puncta using Fiji following immunohistochemistry (Figure 8, A and B). The area occupied by

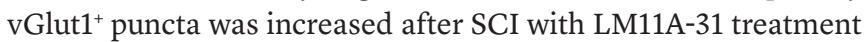
compared with the vehicle-treated control, suggesting that blocking proNGF action through p75 facilitated the increase in glutamatergic inputs. The vGlut1 inputs to $\mathrm{TH}^{+}$neurons also increased, 
A
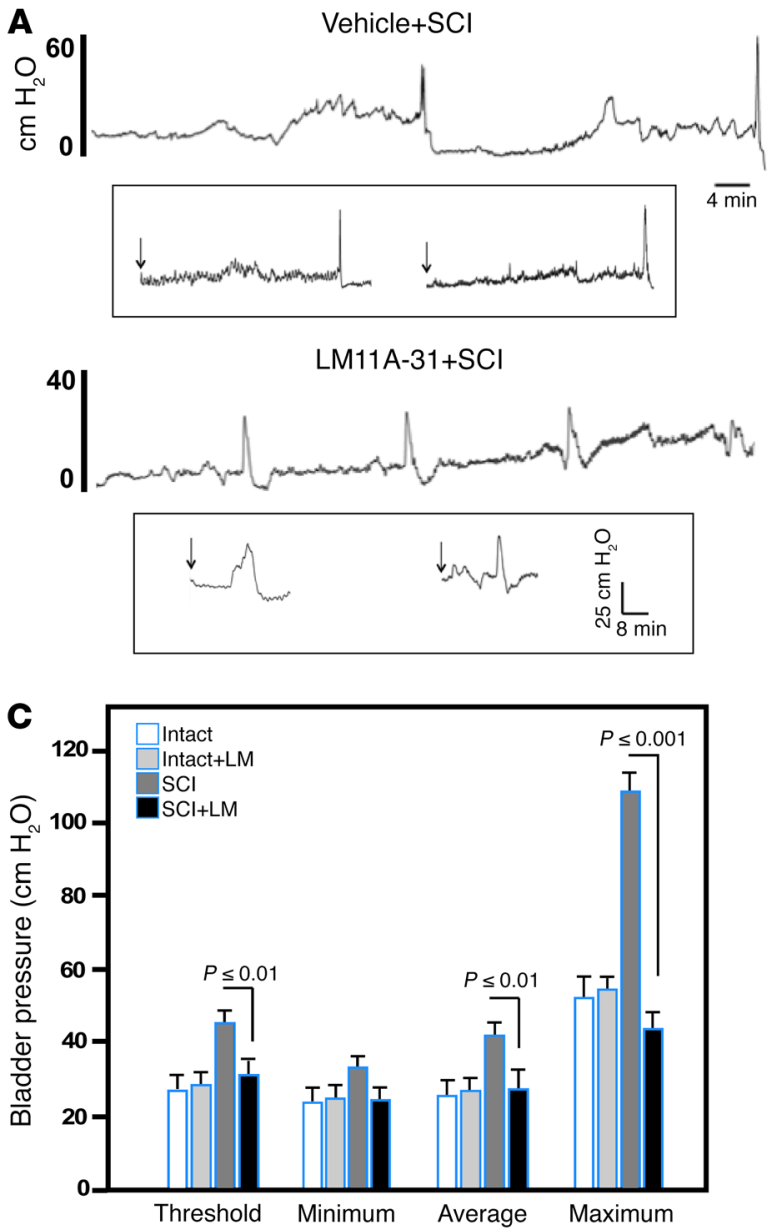

D
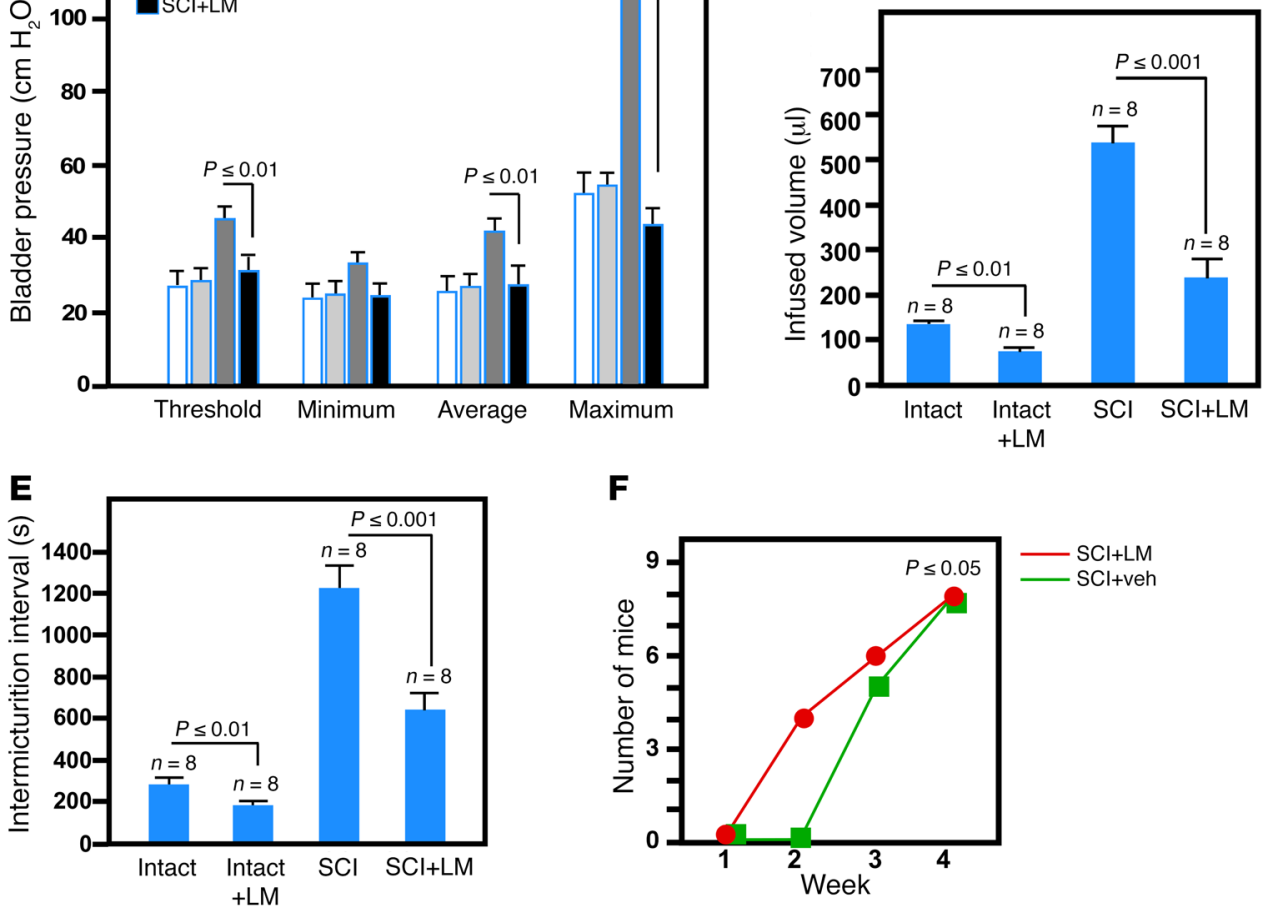

B

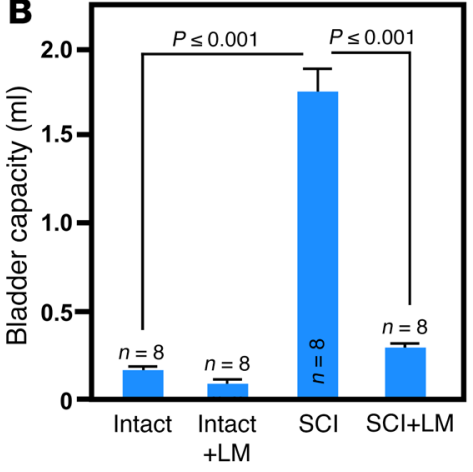

$+\mathrm{LM}$
$\mathbf{F}$

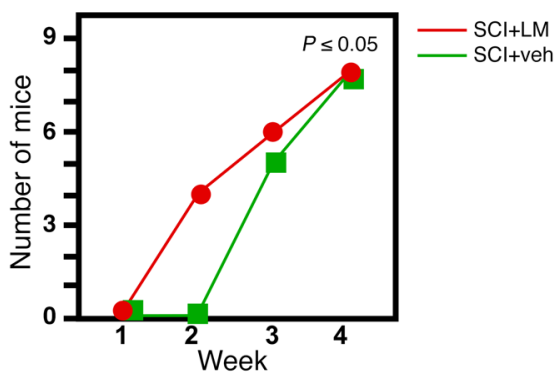

Figure 6. Systemic LM11A-31 greatly improves bladder function after $\mathrm{SC}$ in mice. (A) Representative bladder function recordings in conscious, unrestrained vehicle- or LM11A-31-treated mice with continuous intravesical instillation of saline with an open outlet at 28 days after injury. Upper recordings were from $\mathrm{SCl}$ mice that were treated with vehicle, while lower ones were from $\mathrm{SCI}$ mice that were treated with $100 \mathrm{mg} / \mathrm{kg}$ LM11A-31. Boxed areas: Representative single-fill cystometry recordings (arrow, infusion start) in conscious, unrestrained vehicle- or LM11A-31-treated mice from 2 different experiments. (B) After SCl, LM11A-31 significantly reduced bladder capacity, reaching that of spinalintact mice. (C) Micturition pressures (threshold, average, maximum) were significantly reduced compared with those in vehicle-treated mice. ( $D$ and E) Infused volume and intermicturition intervals were reduced compared with those in vehicle-treated mice in both spinal-intact and SCl groups. (F) Greater numbers of mice treated with LM11A-31 recovered automatic micturition significantly earlier than vehicletreated mice after $\mathrm{SCl}$. Time (days) to recovery of automatic micturition for 2 groups was compared using a 2-tailed unpaired Student's $t$ test. $P \leq 0.05$ was considered statistically significant. Comparisons of cystometric parameters among groups (B-E) were made using ANOVA. When $F$ ratios exceeded the adjusted critical value $(P \leq 0.0125)$, Bonferroni's multiple-comparisons test was used to compare means among groups. as the area occupied with vGlut1 $1^{+}$puncta among $\mathrm{TH}^{+}$fibers was increased within the DCM with LM11A-31 administration (Figure $8 \mathrm{C})$. These results suggest that activation of proNGF/p75 signaling after SCI contributes to the reflex circuit that is responsible for detrusor-sphincter dyssynergia and/or detrusor hyperreflexia, and blocking their action is a way to alleviate bladder dysfunction that accompanies SCI.

\section{Discussion}

Here, we report that proNGF/p75 signaling plays multiple roles in micturition after SCI. Overall, proNGF/p75 signaling is detrimental to bladder function following SCI: with systemic administration of LM11A-31 that blocks proNGF action through p75, bladder capacity and pressure dropped to an almost normal level, hyperre- flexia was significantly attenuated, and automatic micturition was acquired weeks earlier than in the vehicle controls. The improvement was in part attributed to an increased afferent glutamatergic synaptic input onto newly emerged $\mathrm{TH}^{+}$neurons in the DCM and their projections. Surprisingly, p75 also plays a role in modulating frequency of voiding in the naive bladder without SCI, but its effect is opposite to SCI conditions, as selective deletion of p75 in the urothelium resulted in hyperreflexia. We demonstrate that the mechanism by which urothelial p75 influences voiding frequency involves modulating TrpV4 activity: addition of proNGF to primary urothelial cells led to attenuation of TrpV4 activity. Since the inhibition was in response to proNGF, we surmise that urinary proNGF is present normally but below our detection limit of $78-156 \mathrm{pg} / \mathrm{ml}$. After SCI, however, $\mathrm{p} 75^{\wedge}-\mathrm{UP} 3 \mathrm{a}$ mice exhibited 
A

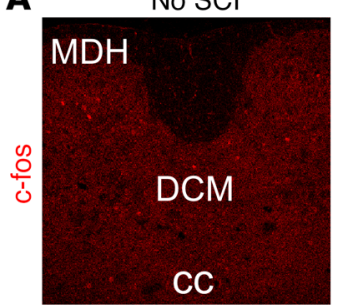

C
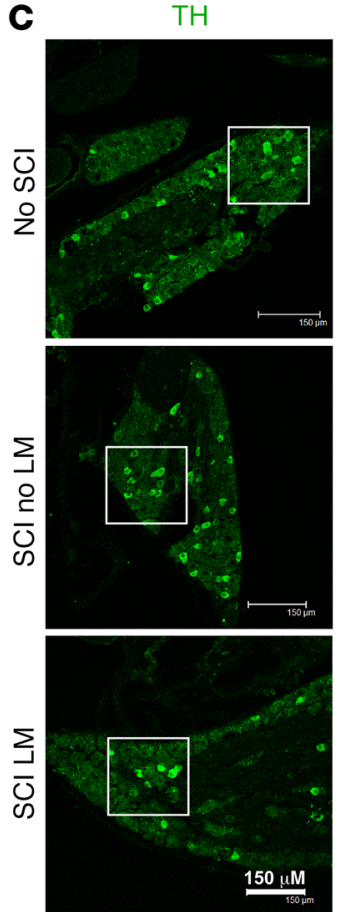

D
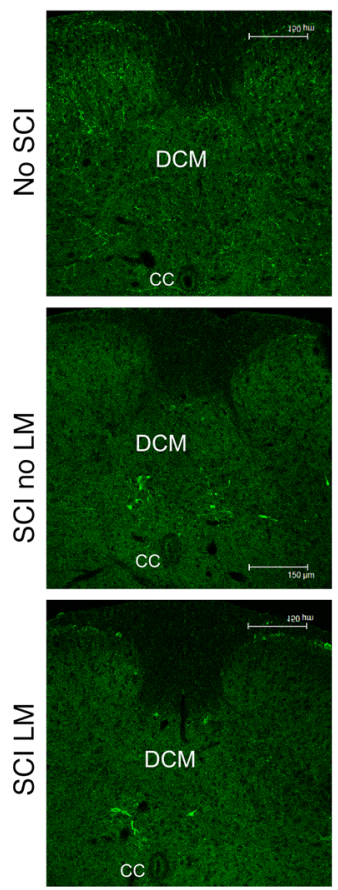

Vehicle

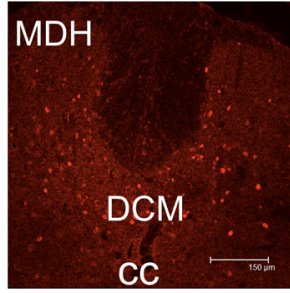

p75
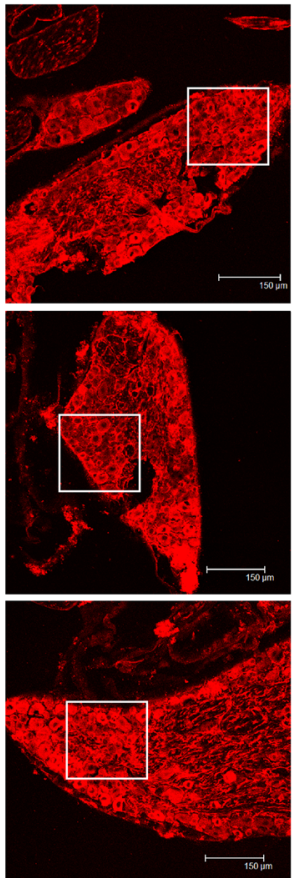

p75
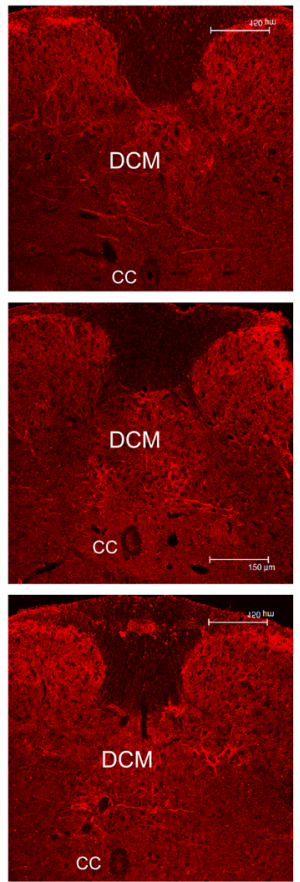

LM11A-31

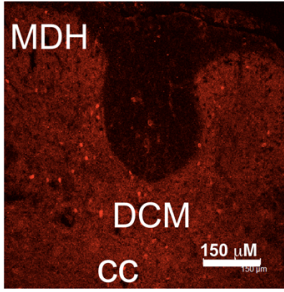

TH/p75
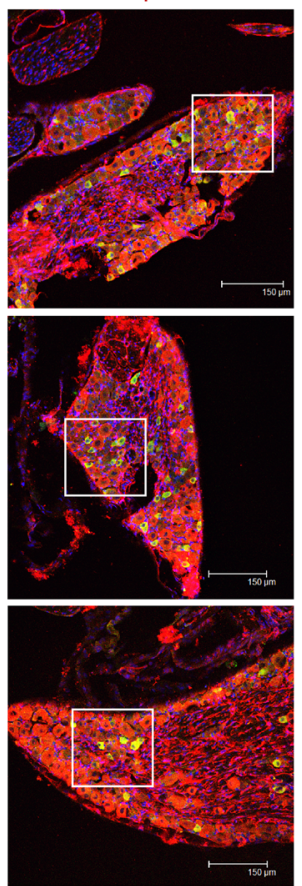

$\mathrm{TH} / \mathrm{p} 75$
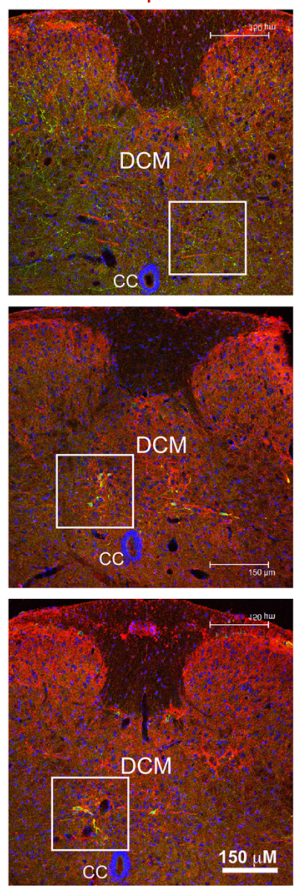

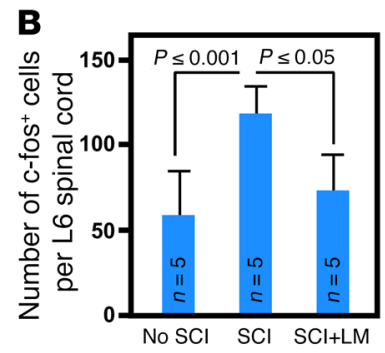

$\mathrm{TH} / \mathrm{p} 75$
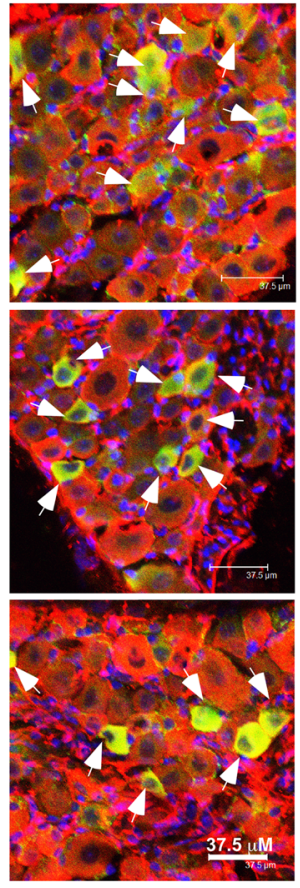

$\mathrm{TH} / \mathrm{p} 75$
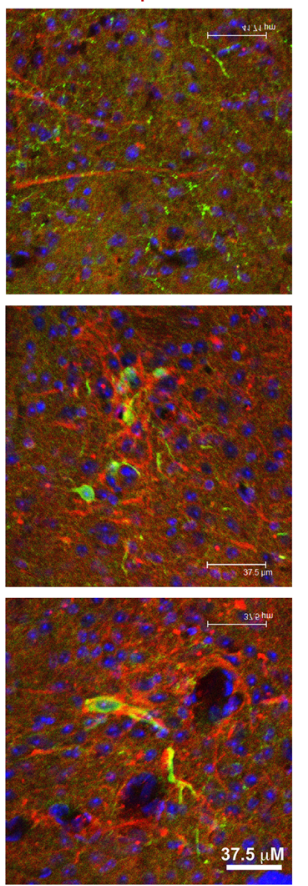

Figure 7. Colocalization of tyrosine hydroxylase among p75+ neurons in DRG and L6/S1 spinal cords after SCI. (A) LM11A-31 reduced the number of $\mathrm{c}-\mathrm{fos}^{+}$ cells in L6 spinal cords after SCI. DCM, dorsal commissure; cc, central canal; $\mathrm{MDH}$, medial dorsal horn. Scale bar: 150 $\mu \mathrm{m}$. (B) Quantification of A. Comparisons among groups were made using 1 -way ANOVA $(P \leq 0.001)$, and pairwise comparisons were made by Tukey's multiple-comparisons test. (C) p75 is expressed among tyrosine hydroxylasepositive $\left(\mathrm{TH}^{+}\right)$sensory neurons in L6/S1 DRG. DRGs were processed for double immunohistochemistry for TH and $\mathrm{p} 75$. Scale bars: $150 \mu \mathrm{m}$. The boxed areas are shown in the far right column; scale bars: $37.5 \mu \mathrm{m}$. (D) Colocalization of TH and p75 in fibers and neuronal soma in the DCM of L6/S1 spinal cords. Scale bars: $150 \mu \mathrm{m}$. The boxed areas are shown in the far right column; scale bars: $37.5 \mu \mathrm{m}$. Experiments were performed 3 times, and representative ones are shown. 
A
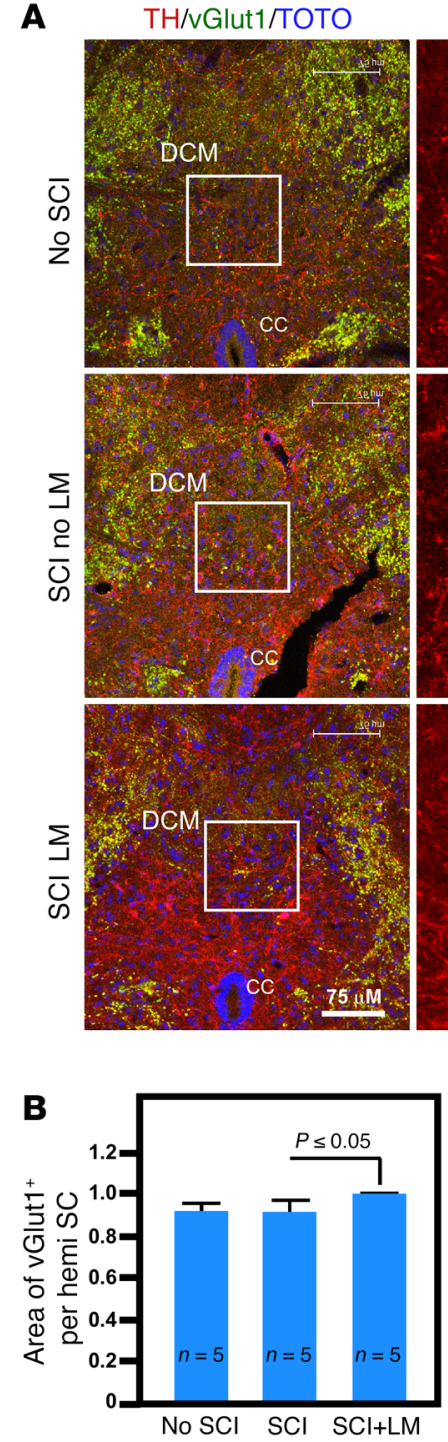

$\mathrm{TH}$
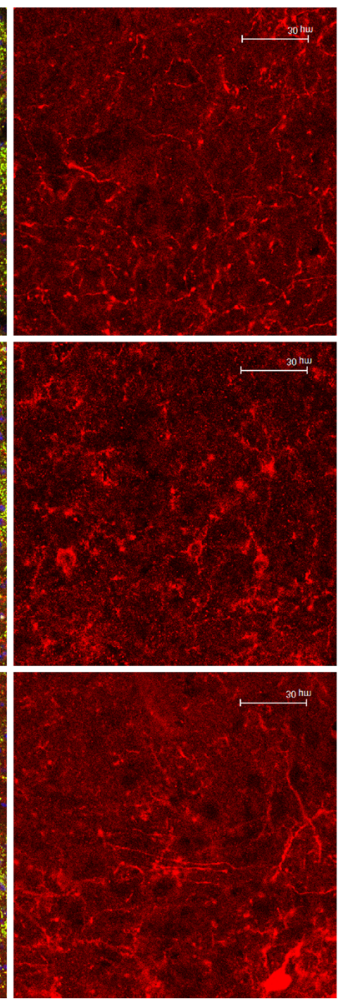

vGlut1
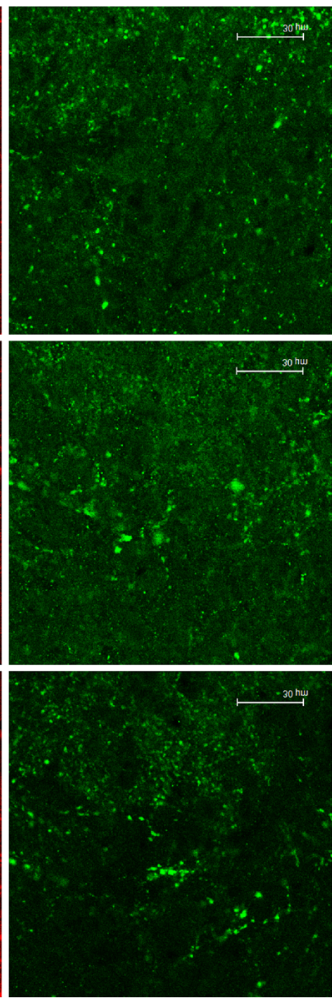

TH/vGlut1/TOTO
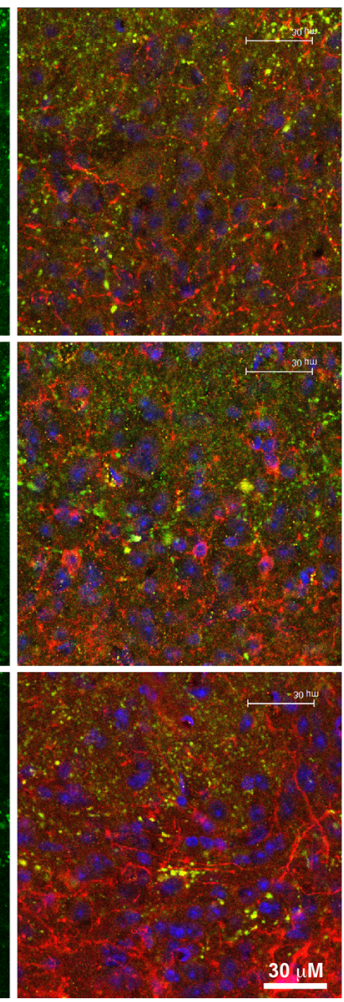

Figure 8. Systemic LM11A-31 increases glutamatergic inputs after $\mathbf{S C l}$ in mice. (A) Representative images of TH and vGlut1 immunostaining of L6/S1 spinal cords. Scale bars: $75 \mu \mathrm{m}$. The boxed areas are shown in 3 columns to the right; scale bars: $30 \mu \mathrm{m}$. (B) LM11A-31 increased the vGlut1+ synaptic puncta. Comparisons among groups were made using 1-way ANOVA, and pairwise comparisons were made by Tukey's multiplecomparisons test. (C) LM11A-31 increased glutamatergic inputs to $\mathrm{TH}^{+}$fibers. Comparisons among groups were made using 1-way ANOVA $(P \leq 0.05)$, and pairwise comparisons were made by Tukey's multiple-comparisons test.

worsened micturition behavior compared with the control. We attribute the phenotype to inhibition of urothelial reorganization that is initiated by apoptosis of umbrella cells that depend on urinary proNGF binding to p75 on umbrella cells: proNGF antibody and LM11A-31 instillation immediately after SCI completely blocked umbrella cell apoptosis as well as ensuing urothelial hyperplasia and detrusor hypertrophy. This unexpected result suggests that the usual turnover of the urothelium that is initiated by the loss of lumen-lining umbrella cells plays a protective role for overall micturition after SCI, perhaps by engaging important ion channels and receptors whose activities change with SCI in the urothelium (1). A rise in urinary proNGF is then a critical signal facilitating regeneration of the urothelium. It is unclear at the moment to what extent proNGF/p75 signaling in the urothelium contributed to overall bladder function after SCI, which requires further study. Regardless, it appears that the role that proNGF/ p75 signaling plays in the CNS to inhibit bladder function after SCI overrides any peripheral contribution.

It was reported that acute subcutaneous delivery of capsaicin improved bladder function after SCI (28). Since capsaicin has no effect on uninjured animals, it was hypothesized that normally "silent" C-fiber bladder afferents acquired mechanosensitivity and initiated automatic micturition (31). Although the mechanism by which capsaicin improves bladder function is still unclear, these results highlight the fact that sensory afferent projections are likely to be involved. Indeed, we found that excitatory glutamatergic inputs to the L6/S1 spinal cord are increased with LM11A-31 administration, and also onto $\mathrm{TH}^{+}$cell bodies and fiber projections. These $\mathrm{TH}^{+}$neurons appear to participate in bladder reflex circuitry after SCI, since transneuronal labeling from the bladder wall labeled $\mathrm{TH}^{+}$cells in similar regions to those identified in the current study (30). In addition, 6-hydroxydopamine treatment not only reduced the number of these $\mathrm{TH}^{+}$neurons but also reduced voiding efficiency (30). Although the characteristics of these $\mathrm{TH}^{+}$neurons need further study, this suggests that these neurons underlie the emergence of automatic micturition after SCI. A complete spinal cord transection injury, rostral to the lumbosacral spinal cord (i.e., upper motoneuron injury), irreversibly impairs urinary bladder function by affecting storage and elimination neural circuitry. Following SCI, detrusor sphincter dyssynergia (DSD) 
and detrusor hyperreflexia (DH) eventually develop, resulting in highly inefficient voiding with high intravesical pressures and large residual urine volumes as the bladder tries to empty against a closed outlet. It has been demonstrated that neutralizing NGF in the lumbosacral spinal cord with NGF-specific antibody suppressed the development of DSD (5) and reduced DH (32). Since it is proNGF and not mature NGF that is released in the cord after SCI (23), it is likely that it was in fact proNGF rather than mature NGF that was neutralized by the antibody, which would have neutralized proNGF as well, owing to shared mature NGF domain. If that were the case, it would suggest that p75 action was being blocked by the NGF neutralization in the spinal cord, which is in line with LM11A-31-mediated improvement observed. We thus surmise that proNGF/p75 signaling may be involved in formation of DSD and that blocking their action with a small-molecule drug, such as LM11A-31, is a viable therapeutic strategy.

\section{Methods}

Human and animal studies. All procedures for human sample collection were approved by the Institutional Review Board at Ohio State University, and followed Department of Health and Human Services and FDA regulations. Written informed consent was received from participants prior to inclusion in the study. All animal studies were approved by the Institutional Animal Care and Use Committee of the Ohio State University the University of Vermont, and followed NIH guidelines for the proper use and care of laboratory animals.

Global and conditional 75 knockout mice. A congenic C57BL/6 line that carries a mutation in exon 3 of the 75 gene was used for the study (33). Its genotype was determined by PCR analyses of tail DNA (34). Conditional p75 knockout mice in C57BL/6 background were generated by crossing of $\mathrm{p} 75^{\mathrm{fl} / \mathrm{ll}}$ mice with a UP3a-GFP/Cre/ERT2 line also in C57BL/6 background $(16,17)$. The experimental line, UP3a-CreGFP/ $\mathrm{ERT}^{+/-}$p $75^{\mathrm{fl} / \mathrm{fl}}$, and a control line, UP3a-CreGFP/ERT2 ${ }^{+/-}$p $75^{-/-}$, are referred to as $\mathrm{p} 75^{\wedge}$-UP3a and $\mathrm{p} 75^{\mathrm{c}-U P 3 a}$, respectively, for brevity. To delete 75 from the urothelium, adult mice were treated with $160 \mathrm{mg} /$ $\mathrm{kg}$ tamoxifen for 5 days via daily oral gavage, and subjected to experimentation 10-14 days later. Except for cystometric studies, which used only female mice, both males and females that were older than 2-3 months were used for the study.

Genomic PCR of the urothelium following tamoxifen administration. At 1-2 weeks after tamoxifen administration, bladders were isolated from $\mathrm{p} 75^{\mathrm{c}}$-UP3a and $\mathrm{p} 75^{\Delta}$-UP3a mice, and placed onto a sterile wax plate in MEM media. The bladders were cut longitudinally and stretched flat with the urothelium side up using $0.1 \mathrm{~mm} \times 1 \mathrm{~cm}$ pins, and incubated with $2 \mathrm{mg} / \mathrm{ml}$ dispase in MEM for 30 minutes at $37^{\circ} \mathrm{C}$. The urothelial layer was then gently scraped with a spatula and collected to extract genomic DNA. The rest of the bladder lacking the urothelium was also collected as a control. PCR reactions on isolated genomic DNA were performed as described in ref. 17.

SCI and postoperative care. Spinal cord transections were performed under isoflurane anesthesia ( $2 \%-3 \%$ in a balance of oxygen for induction and $1 \%-2 \%$ for maintenance) in adult female mice. A laminectomy was performed at the T8-T10 level followed by complete transection of the dura and spinal cord using iris scissors under visual guidance with a surgical microscope. DRGs, rami, and rami communicantes were not transected. We confirmed complete transection of the spinal cord by observing the rostral and caudal cut ends of the spinal cord retract under the surgical microscope. The space between the retracted ends of the spinal cord was packed with sterile Gelfoam (Pharmacia and Upjohn), and the incision was sutured. Carprofen (5 $\mathrm{mg} / \mathrm{kg}$, s.c.) was given at the time of surgery and for 2 days after injury at 24-hour intervals. The T9 epicenter was chosen to avoid the rostral lumbar and caudal lumbosacral spinal cord, which are involved in the storage and elimination phases of the voiding reflex, respectively. Our experience indicates that a T9 epicenter injury creates a reproducible upper motoneuron injury that is useful to evaluate the recovery of urinary bladder function. The complete transection model of SCI is useful to study recovery of urinary bladder function without the possibility of plasticity processes bypassing the lesion. LM11A-31 was orally delivered at $100 \mathrm{mg} / \mathrm{kg}$ beginning 4 hours after injury, and once daily thereafter for 4 weeks. Urinary bladders were manually expressed 2-3 times a day. We used female mice because manual expression of bladder (Credé maneuver) is more easily accomplished because of the shorter and wider urethra in female compared with male mice. We recorded presence or absence of automatic micturition days after injury at the first daily manual expression. Automatic micturition is defined as the period following spinal shock with the emergence of a micturition reflex at the spinal level and the appearance of spontaneous, involuntary bladder contractions. Thus, some function is restored although voiding frequency and volume are altered.

Sham animals (laminectomy, spinal cord intact) were not subjected to any further manipulation. For contusion injuries, an Infinite Horizon Impactor (Precision Systems and Instrumentation LLC) was used at a controlled force-defined impact at $50 \mathrm{kdyn}$ at the thoracic 9 position as previously described (11). We used 2 different models of SCI (contusion injury and complete transection injury) to determine the generalizability of proNGF release in the urine. Although a preclinical contusion injury may have more translational relevance, we used a more severe (complete transection) model, because it allows the evaluation of bladder function after injury without the possibility of plasticity processes bypassing the lesion.

$S C I$ in rats. Complete transection was performed at $\mathrm{T} 9$ as previously described under isoflurane anesthesia (35). Briefly, after a T8T10 laminectomy, the dura and spinal cord were cut with scissors and a sterile sponge was placed between the severed ends of the spinal cord. The overlying muscle and skin were sutured and maintained at $23^{\circ} \mathrm{C}-25.5^{\circ} \mathrm{C}$. To prevent any infection, mice were treated with daily subcutaneous dosing of $100 \mathrm{mg} / \mathrm{kg}$ ampicillin for 10-14 days.

Collection of human urine. Urine samples were collected from 2 individuals, after placement of Foley catheters, frozen immediately, and stored at $-80^{\circ} \mathrm{C}$ until analyses. Patient 1 was a 71 -year-old male with a C6-level American Spinal Injury Association Impairment Scale (AIS) C injury following a motorcycle crash, with urine collected at 3 hours after injury. Patient 2 was a 44 -year-old male with a C5-level AIS D injury following a fall from more than 20 feet, with urine collected at 6 hours after injury. The control urine was from a healthy male with no injury.

Implantation of intravesical catheter for conscious cystometry. A lower midline abdominal incision was performed under general anesthesia and secured in place with a 6-0 nylon purse string suture at 14 days (p75 ${ }^{\perp}$-UP3a and $\mathrm{p} 75^{\mathrm{c}}$-UP3a) or 28 days after injury (LM11A-31 and vehicle). The distal end of the tubing was sealed, tunneled subcutaneously, and externalized at the back of the neck, out of the animal's reach. Abdominal and neck incisions were closed with 4-0 nylon suture. Animals were maintained for 48 hours to ensure complete recovery from 
the surgery prior to cystometry. Cystometry in conscious mice was performed according to previously published methods (36). Animals were placed unrestrained in recording cages with a balance and pan for urine collection and measurement placed below. In conscious mice, roomtemperature saline was infused into the urinary bladder via a catheter placed in the bladder dome. Saline solution was infused at room temperature into the bladder at a rate of $25 \mu \mathrm{l} / \mathrm{min}$ to elicit repetitive bladder contractions. Slow-fill (10 $\mu \mathrm{l} / \mathrm{min})$, single cystometry cycles were also recorded in some animals. At least 6 reproducible micturition cycles were recorded after the initial stabilization period of 25-30 minutes. The following cystometric parameters were recorded in each animal: baseline pressure (pressure at the beginning of the bladder filling), threshold pressure (bladder pressure immediately prior to micturition), peak micturition pressure (peak intravesical pressure during voiding), intermicturition interval (time between micturition events), infused volume, and void volume (VV). After VVs were collected, the infusion was stopped and residual volume (RV) was determined: residual saline was withdrawn through the intravesical catheter. Bladder capacity (BC) was calculated as the sum of VV and RV. Based on these values, voiding efficiency $(\%)=[(\mathrm{VV} / \mathrm{BC}) \times 100]$ was estimated. Two hours after saline infusion, animals were euthanized via transcardial perfusion, and the lumbosacral spinal cord and DRGs were harvested for immunohistochemistry studies. Experiments were conducted at similar times of the day to avoid the possibility that circadian variations were responsible for changes in bladder measurements. An individual blinded to genotype or treatment analyzed the cystometric data; groups were decoded after data analysis.

Intravesical instillation. For intravesical instillation of $100 \mathrm{mg} /$ $\mathrm{kg}$ of LM11A-31 and proNGF blocking antibody that was diluted 1:20 in sterile saline, lubricated PE10 tubing that was connected to a 1-ml syringe via a 30-gauge Hamilton needle was gently inserted into the bladder through the urethra of operated female mice immediately after SCI. Reagents were slowly instilled into the bladder in $100 \mu \mathrm{l}$ volume.

Scanning electron microscopy of the bladder surface. Mice were perfused transcardially with $2.5 \%$ glutaraldehyde in $0.1 \mathrm{M}$ phosphate buffer ( $\mathrm{pH}$ 7.2). Bladders were dissected, placed in fixative at $4^{\circ} \mathrm{C}$ for 72 hours, and then rinsed in $0.1 \mathrm{M}$ Na phosphate buffer. The tissues were subjected to a sequential dehydration procedure that was followed by hexamethyldisilazane drying. Samples were then placed into the chamber of a sputter coater and coated with a film of gold/palladium before examination using FEI Nova NanoSEM 400 at the Campus Microscopy \& Imaging Facility at Ohio State University.

Immunohistochemistry. Mice were transcardially perfused with $3 \%$ paraformaldehyde in $0.1 \mathrm{M}$ phosphate buffer $(\mathrm{pH} 7.2)$; bladders and spinal cords were isolated and postfixed in the same fixative for an additional 2 hours prior to overnight cryopreservation in $20 \%$ sucrose in $0.1 \mathrm{M}$ phosphate buffer. The bladders were then filled with OCT compound, frozen on dry ice, cut at $30 \mu \mathrm{m}$ thickness from the dome toward the urethra, and mounted on glass slides coated with $0.15 \%$ gelatin and $0.1 \mathrm{mg} / \mathrm{ml}$ poly-D-lysine. Spinal cords corresponding to L6 and S1 were dissected using L6 and S1 DRGs as guide, mounted in OCT compound, and cryosectioned at $25 \mu \mathrm{m}$ thickness. For immunohistochemistry, sections were incubated for 2 hours at room temperature in blocking solution, which contained $0.3 \%$ Triton X-100, 5\% BSA, and $5 \%$ goat, $5 \%$ horse serum in $1 \times$ Tris-buffered saline (TBS). Primary antibodies were applied overnight at room temperature in a solution containing $0.1 \%$ Triton $\mathrm{X}-100,1 \%$ BSA, $1 \%$ goat, $1 \%$ horse serum, $1 \times$ TBS, and $0.02 \% \mathrm{Na}$ azide. Antibodies used for immunohistochemistry include p75 (9650, a gift from Moses Chao, New York University, New York, New York, USA), pan-uroplakin (AUN, a gift from Tung-Tien Sun, New York University) as a marker for umbrella cells, vGlut1 (a gift from Julia Kaltschmidt, Stanford University, Stanford, California, USA), c-fos (2250, Cell Signaling Technology), NGF (H-20, Santa Cruz Biotechnology), phospho-histone 3 (53348, Cell Signaling Technology), laminin (Abcam), BrdU (555627, BD Biosciences), and tyrosine hydroxylase (2017-THSHP, PhosphoSolutions, and MAB318, Millipore). Slides were counterstained with TOTO-3 for nuclei (Invitrogen), mounted in Slow Fade Diamond Antifade Mountant (Invitrogen), and imaged using a Leica TCS SL confocal microscope.

Quantification of immunofluorescence images. To quantify TH and vGlut1 signals, the images from dorsal and ventral spinal cord hemispheres were first merged using Fiji (https://fiji.sc). The merged images were thresholded from 1 to 255 and processed to binary mode. The gray matter areas were selected, and signals within the selected area were measured. The pixels within the area were calculated by division of the RawIntDen by the maximum, 255, and the data were plotted after division of the pixels by the area of each sample. For quantifying TH and vGlut1 overlapped signals, after each image was made into binary, the overlapped signals were identified by the multiply function of Fiji.

Quantification of c-fos ${ }^{+}$cells. Every ninth section of the spinal cords was processed for c-fos staining, and the number of positive cells was quantified in the DCM areas.

Immunoprecipitation and Western blotting. Mouse bladders were separated into the detrusor muscle and urothelium plus lamina propria, and snap-frozen in liquid $\mathrm{N}_{2}$. For protein extraction, tissues were pulverized, and subsequently homogenized in RIPA buffer containing $1 \%$ NP-40, 0.5\% deoxycholate, $150 \mathrm{mM} \mathrm{NaCl}, 50 \mathrm{mM}$ Tris (pH 8.0), $2 \mathrm{mM}$ EDTA, plus $1 \mathrm{mM}$ sodium orthovanadate, $25 \mathrm{mM} \mathrm{NaF}, 10 \mathrm{mg} / \mathrm{ml}$ leupeptin, $10 \mathrm{mg} / \mathrm{ml}$ aprotinin, and $2 \mathrm{mM}$ PMSF. The procedures for Western blotting were as previously described (37). The antibodies used in the study include p75 (9650, detecting extracellular domain), p75 (BC, detecting intracellular domain; a gift from Bruce Carter, Vanderbilt University, Nashville, Tennessee, USA), proNGF (ANT005, Alomone, and S-080-100, Biosensis), NGF (SC-548, Santa Cruz Biotechnology), pan-Trk (SC-11, Santa Cruz Biotechnology), $\alpha$-actin (SC-32251, Santa Cruz Biotechnology), and Uroplakin II (SC-15178, Santa Cruz Biotechnology). For immunoprecipitation of proNGF, dialyzed urine samples were incubated overnight at $4^{\circ} \mathrm{C}$ on a rotator with $1 \mathrm{mg} / \mathrm{ml}$ of control or 27/21 anti-mouse antibody (a gift from Yves Barde, Cardiff University, Cardiff, United Kingdom). The immune complexes were pulled down with protein A/G beads, washed in the RIPA buffer, and subjected to SDS-PAGE. The blots were probed with the indicated NGF antibodies. See complete unedited blots in the supplemental material.

TUNEL labeling and quantification. For TUNEL assays, the sections were treated with $0.1 \%$ citric acid and $0.1 \%$ Triton $\mathrm{X}-100$ before incubation with TUNEL solutions at $37^{\circ} \mathrm{C}$ for 1 hour. TUNEL reaction was performed using a Roche TUNEL kit. For quantification, every third section was processed and counted.

ELISA assays. ELISA kits for detecting mouse mature and proNGF as well as human proNGF were purchased from Biosensis. Both proNGF ELISA kits are specific to proNGF and do not detect mature NGF. The ELISA kit for human mature NGF was purchased from SigmaAldrich. The assays were performed according to the manufacturers' instructions using diluted urine samples. 
Calcium imaging of primary mouse urothelial cultures. Primary mouse urothelial cultures were obtained as previously described (21, 38) but with modifications. The stretched adult bladders were incubated with $2 \mathrm{mg} / \mathrm{ml}$ dispase II in MEM for 30 minutes at $37^{\circ} \mathrm{C}$, and the urothelial cells were scraped and gently resuspended before plating in CnT-PR medium (CELLnTEC) on plastic dishes that were coated with Coating Matrix Kit (Thermo Fisher Scientific). At 3-5 days in vitro, cells were washed 3 times with calcium imaging buffer (CIB), which contained $105 \mathrm{mM} \mathrm{NaCl}, 3 \mathrm{mM} \mathrm{KCl}, 2.5 \mathrm{mM} \mathrm{CaCl}_{2}, 0.6 \mathrm{mM}$ $\mathrm{MgCl}_{2}, 10 \mathrm{mM}$ HEPES, $1.2 \mathrm{mM} \mathrm{NaHCO}, 100 \mathrm{mM}$ mannitol, and 10 $\mathrm{mM}$ glucose at $\mathrm{pH} 7.45$ (39), and loaded with $3 \mu \mathrm{M}$ Fluo-4 AM in the presence of $0.02 \%$ Pluronic F-127 (Thermo Fisher Scientific). After 30 minutes of incubation at $37^{\circ} \mathrm{C}$, cells were again washed 3 times with $\mathrm{CIB}$, and incubated for an additional 30 minutes at $37^{\circ} \mathrm{C}$ for de-esterification. For live imaging, dishes were maintained at $37^{\circ} \mathrm{C}$ using a temperature-controlled imaging setup that was associated with a Zeiss Axio Observer Inverted Z1 equipped with Zen 2 software. Following 3 minutes of initial imaging, the endogenous TrpV4 was

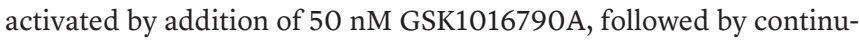
ous imaging for 30 additional minutes. For NGF addition, cells were incubated with $10 \mathrm{ng} / \mathrm{ml}$ of recombinant pro-NGF (Alomone) for 30 minutes after Fluo-4 AM loading, imaged for 3 minutes, then treated with $50 \mathrm{nM}$ GSK1016790A followed by 30 minutes of imaging. The calcium imaging data were analyzed using MATLAB (MathWorks) and Prism (GraphPad Software).

Cell-surface biotinylation assays. Before surface biotinylation assays, 293T cells that were transfected with FLAG-TrpV4 (40), HA-p75, and sortilin were incubated with $10 \mathrm{ng} / \mathrm{ml}$ of proNGF at $37^{\circ} \mathrm{C}$ for 30 minutes, then with $50 \mathrm{nM}$ GSK1016790A for the indicated times. At the end of incubation, cells were washed 3 times with icecold PBS plus $0.1 \%$ glucose, and subjected to biotinylation using EZLink Sulfo-NHS-SS-Biotin (Pierce) for 30 minutes with a gentle shaking on ice. The biotinylation reaction was stopped by addition of an equal volume of $50 \mathrm{mM}$ Tris ( $\mathrm{pH}$ 7.4), and the resulting lysates were subjected to NeutrAvidin pull-down followed by Western blotting for FLAG to detect TrpV4 (Sigma-Aldrich). To detect p75, the blot was stripped and reprobed for p75.
Statistics. Data are presented as the mean \pm SEM. GraphPad Prism 7 or SPSS (IBM) software was used to perform the statistical analysis. ProNGF protein levels and the number of TUNEL counts were compared using repeated measures of 1-way ANOVA after GreenhouseGeisser corrections ( $P \leq 0.05$ and 0.001$)$, with pairwise comparisons using Bonferroni test. Multiple comparisons were performed using 1-way ANOVA ( $P \leq 0.05)$ with Tukey's multiple-comparisons test, while paired Student's $t$ tests were used to determine 2-tailed significance. For cystometric data analyses involving comparisons of more than 2 groups, 1-way ANOVAs were used according to the experimental design with Bonferroni's multiple-comparisons tests. $P$ values less than or equal to 0.0125 were considered statistically significant.

\section{Author contributions}

JCR, KT, SEM, AS, NS, TW, NG, SD, YI, IZ, AJK, and HFF conducted experiments and acquired and analyzed data. US provided critical reagents. MY and WCDG provided critical consultation. MAV and SOY analyzed data and wrote the manuscript.

\section{Acknowledgments}

This work was supported by grants from the National Institute of Diabetes and Digestive and Kidney Diseases (NIDDK; R01DK051369 and R01DK060481) and the National Institute of Neurological Disorders and Stroke (NINDS; R25NS090623) to M.A. Vizzard; and from the NIDDK (R56DK104137 and R21NS098362), the Lone Star Paralysis Foundation, the Alzheimer's Drug Discovery Foundation, and the NINDS (5P30NS045758) to S.O. Yoon. We thank Jamie Jontes for help with MATLAB analyses and Paula Monsma for Fiji-based quantification. We also thank Jerry Silver and Bruce Carter for reading the manuscript.

Address correspondence to: Margaret A. Vizzard, D405A Given Research Building, 89 Beaumont Avenue, Larner College of Medicine, Burlington, Vermont 05405, USA. Phone: 802.656.3209; Email: Margaret.Vizzard@uvm.edu. Or to: Sung Ok Yoon, 184 Rightmire Hall, Ohio State University, 1060 Carmack Road, Columbus, Ohio 43210, USA. Phone: 614.292.8542; Email: sung.yoon@osumc.edu.
1. Fowler CJ, Griffiths D, de Groat WC. The neural control of micturition. Nat Rev Neurosci. 2008;9(6):453-466.

2. de Groat WC, Yoshimura N. Mechanisms underlying the recovery of lower urinary tract function following spinal cord injury. Prog Brain Res. 2006;152:59-84.

3. Ochodnicky P, Cruz CD, Yoshimura N, Cruz F. Neurotrophins as regulators of urinary bladder function. Nat Rev Urol. 2012;9(11):628-637.

4. Ochodnický P, Cruz CD, Yoshimura N, Michel MC. Nerve growth factor in bladder dysfunction: contributing factor, biomarker, and therapeutic target. Neurourol Urodyn. 2011;30(7):1227-1241.

5 . Seki S, et al. Suppression of detrusor-sphincter dyssynergia by immunoneutralization of nerve growth factor in lumbosacral spinal cord in spinal cord injured rats. JUrol. 2004;171(1):478-482.

6. Hu VY, et al. Decrease in bladder overactivity with REN1820 in rats with cyclophosphamide induced cystitis. J Urol. 2005;173(3):1016-1021.

7. Teng KK, Felice S, Kim T, Hempstead BL.
Understanding proneurotrophin actions: recent advances and challenges. Dev Neurobiol. 2010;70(5):350-359.

8. Klinger MB, Girard B, Vizzard MA. p75NTR expression in rat urinary bladder sensory neurons and spinal cord with cyclophosphamide-induced cystitis. J Comp Neurol. 2008;507(3):1379-1392.

9. Klinger MB, Vizzard MA. Role of p75NTR in female rat urinary bladder with cyclophosphamide-induced cystitis. Am J Physiol Renal Physiol. 2008;295(6):F1778-F1789.

10. Massa SM, et al. Small, nonpeptide p75NTR ligands induce survival signaling and inhibit proNGF-induced death. J Neurosci. 2006;26(20):5288-5300.

11. Tep C, et al. Oral administration of a small molecule targeted to block proNGF binding to p75 promotes myelin sparing and functional recovery after spinal cord injury. J Neurosci. 2013;33(2):397-410.

12. Vizzard MA. Changes in urinary bladder neurotrophic factor mRNA and NGF protein follow- ing urinary bladder dysfunction. Exp Neurol. 2000;161(1):273-284.

13. Apodaca G, Kiss S, Ruiz W, Meyers S, Zeidel M, Birder L. Disruption of bladder epithelium barrier function after spinal cord injury. Am J Physiol Renal Physiol. 2003;284(5):F966-F976.

14. Shin K, et al. Hedgehog/Wnt feedback supports regenerative proliferation of epithelial stem cells in bladder. Nature. 2011;472(7341):110-114.

15. Khandelwal P, Abraham SN, Apodaca G. Cell biology and physiology of the uroepithelium. AmJ Physiol Renal Physiol. 2009;297(6):F1477-F1501.

16. Gandhi D, et al. Retinoid signaling in progenitors controls specification and regeneration of the urothelium. Dev Cell. 2013;26(5):469-482.

17. Bogenmann E, et al. Generation of mice with a conditional allele for the p75(NTR) neurotrophin receptor gene. Genesis. 2011;49(11):862-869.

18. Zvara P, Vizzard MA. Exogenous overexpression of nerve growth factor in the urinary bladder produces bladder overactivity and altered micturition circuitry in the lumbosacral spinal cord. 
BMC Physiol. 2007;7:9.

19. Gevaert T, et al. Deletion of the transient receptor potential cation channel TRPV4 impairs murine bladder voiding. JClin Invest. 2007;117(11):3453-3462.

20. Yu W, Hill WG, Apodaca G, Zeidel ML. Expression and distribution of transient receptor potential (TRP) channels in bladder epithelium. Am J Physiol Renal Physiol. 2011;300(1):F49-F59.

21. Mochizuki T, et al. The TRPV4 cation channel mediates stretch-evoked $\mathrm{Ca}^{2+}$ influx and ATP release in primary urothelial cell cultures. J Biol Chem. 2009;284(32):21257-21264.

22. Jin M, et al. Determinants of TRPV4 activity following selective activation by small molecule agonist GSK1016790A. PLoS One. 2011;6(2):e16713.

23. Beattie MS, et al. ProNGF induces p75-mediated death of oligodendrocytes following spinal cord injury. Neuron. 2002;36(3):375-386.

24. Harrington AW, et al. Secreted proNGF is a pathophysiological death-inducing ligand after adult CNS injury. Proc Natl Acad Sci U S A. 2004;101(16):6226-6230.

25. Sharma N, et al. Long-distance control of synapse assembly by target-derived NGF. Neuron . 2010;67(3):422-434.

26. Birder LA, de Groat WC. Increased c-fos expression in spinal neurons after irritation of the lower urinary tract in the rat. J Neurosci. 1992;12(12):4878-4889.

27. Vizzard MA. Increased expression of spinal cord Fos protein induced by bladder stimulation after spinal cord injury. Am J Physiol Regul Integr Comp Physiol. 2000;279(1):R295-R305.

28. Cheng CL, Ma CP, de Groat WC. Effect of capsaicin on micturition and associated reflexes in chronic spinal rats. Brain Res. 1995;678(1-2):40-48.

29. Li L, et al. The functional organization of cutaneous low-threshold mechanosensory neurons. Cell. 2011;147(7):1615-1627.

30. Hou S, Carson DM, Wu D, Klaw MC, Houlé JD, Tom VJ. Dopamine is produced in the rat spinal cord and regulates micturition reflex after spinal cord injury. Exp Neurol. 2016;285(pt B):136-146.

31. Häbler HJ, Jänig W, Koltzenburg M. Activation of unmyelinated afferent fibres by mechanical stimuli and inflammation of the urinary bladder in the cat. J Physiol (Lond). 1990;425:545-562.

32. Seki S, et al. Immunoneutralization of nerve growth factor in lumbosacral spinal cord reduces bladder hyperreflexia in spinal cord injured rats. JUrol. 2002;168(5):2269-2274.

33. Lee KF, et al. Targeted mutation of the gene encoding the low affinity NGF receptor p75 leads to deficits in the peripheral sensory nervous sys- tem. Cell. 1992;69(5):737-749.

34. Bentley CA, Lee KF. p75 is important for axon growth and schwann cell migration during development. J Neurosci. 2000;20(20):7706-7715.

35. Artim DE, Kullmann FA, Daugherty SL, Bupp E, Edwards CL, de Groat WC. Developmental and spinal cord injury-induced changes in nitric oxide-mediated inhibition in rat urinary bladder. Neurourol Urodyn. 2011;30(8):1666-1674.

36. Schnegelsberg B, et al. Overexpression of NGF in mouse urothelium leads to neuronal hyperinnervation, pelvic sensitivity, and changes in urinary bladder function. Am J Physiol Regul Integr Comp Physiol. 2010;298(3):R534-R547.

37. Harrington AW, Kim JY, Yoon SO. Activation of Rac GTPase by p75 is necessary for c-jun N-terminal kinase-mediated apoptosis. J Neurosci. 2002;22(1):156-166.

38. Birder LA, et al. Altered urinary bladder function in mice lacking the vanilloid receptor TRPV1. Nat Neurosci. 2002;5(9):856-860.

39. Wheeler MA, et al. Genetically targeted magnetic control of the nervous system. Nat Neurosci. 2016;19(5):756-761.

40. Shukla AK, et al. Arresting a transient receptor potential (TRP) channel: beta-arrestin 1 mediates ubiquitination and functional down-regulation of TRPV4. J Biol Chem. 2010;285(39):30115-30125. 